\title{
Base-Free Mizoroki-Heck Reaction Catalysed by Rhodium Complexes
}

\author{
Rémi Martinez, Florina Voica, Jean-Pierre Genet, ${ }^{*}$ and Sylvain \\ Darses* \\ Laboratoire de Synthèse Sélective Organique (UMR 7573, CNRS),Ecole Nationale \\ Supérieure de Chimie de Paris, 11 rue P\&M Curie, 75231 Paris cedex 05 (France) \\ sylvain-darses@enscp.fr,jean-pierre-genet@enscp.fr
}

\section{Supporting information}

\section{General Procedures}

${ }^{1} \mathrm{H}$ NMR and ${ }^{13} \mathrm{C}$ NMR spectra were recorded on a Avance 300 or Avance 400 instruments; chemical shifts $(\delta)$ are reported in ppm relative to $\mathrm{Me}_{4} \mathrm{Si}$; coupling constants $(J)$ are reported in $\mathrm{Hz}$ and refer to apparent peak multiplicities. ${ }^{2} \mathrm{H}$ NMR spectra were recorded on a Bruker Avance 400 instrument at $61.4 \mathrm{MHz}$ using benzene- $d_{6}$ as an internal reference. Mass spectra were determined on a Ribermag instrument. High resolution mass spectra were performed on a Varian MAT311 at the Université Pierre et Marie Curie (Paris). Thin layer chromatography was carried out on silica-gel plates (Merck F254), spots were detected with UV light and revealed with KMnO4 solution. GC analyses were performed on a Hewlett-Packard instrument equipped with a J\&W Scientific DB-1701 capillary column $(15 \mathrm{~m}, \varnothing=$ $0.25 \mu \mathrm{m})$, using an ionization flame detector.

Potassium organotrifluoroborates ${ }^{1}$ were prepared according to published procedures. Dioxane and acetone were distilled from $\mathrm{CaH}_{2}$. Activated alkenes were freshly distilled before use.

Typical procedure for rhodium-catalyzed alkenylation of potassium aryltrifluoroborates: preparation of (butyl 3(4-methoxyphenyl)acrylate (entry 3): To a mixture of potassium trifluoro(4-methoxyphenyl)borate (1 mmol, $214 \mathrm{mg}$ ), chlorobis(ethylene)rhodium(I) dimer $(15 \mu \mathrm{mol}, 5.8 \mathrm{mg})$ and triphenylphosphane (60 $\mu \mathrm{mol}, 15.7 \mathrm{mg})$ in degassed dioxane $(2$ $\mathrm{mL})$ and degassed acetone $(0.5 \mathrm{~mL})$ was added butyl acrylate $(2 \mathrm{mmol}, 288 \mu \mathrm{l})$, under an argon atmosphere. The flask was placed in a preheated oil bath at $80^{\circ} \mathrm{C}$. The mixture was stirred until completion of the reaction (followed by GC analysis). After concentration under reduced pressure, the crude mixture was purified by silica gel chromatography eluting with cyclohexane/ethyl acetate (95:5) to afford $217 \mathrm{mg}$ of analytically pure alkene 3d as a colorless oil (yield 92\%).

1-(3-Bromophenyl)-hex-1-en-3-one 3m (entry 12):

Reaction of potassium 3-bromophenyltrifluoroborate (1 mmol, $263 \mathrm{mg})$ and hex-1-en-3-one (3 mmol, $294 \mathrm{mg})$ afforded $213 \mathrm{mg}$ of enone $3 \mathrm{~m}$ as a pale yellow oil (yield $84 \%)$. GC $\left(50 \mathrm{kPa}, 70^{\circ} \mathrm{C}\right.$ during 1 min then $20^{\circ} \mathrm{C} / \mathrm{min}$ up to $\left.210^{\circ} \mathrm{C}\right): \mathrm{t}_{\mathrm{R}} 10.00$ min. Rf (petroleum ether / ethyl acetate 20/1): 0.24. ${ }^{1} \mathrm{H} \mathrm{NMR}\left(\mathrm{CDCl}_{3}, 300 \mathrm{MHz}\right) \delta=7.69(\mathrm{t}$ app, $J=1.8 \mathrm{~Hz}, 1 \mathrm{H}), 7.46(\mathrm{~d}, J=$ $16.4 \mathrm{~Hz}, 1 \mathrm{H}), 7.44-7.52(\mathrm{~m}, 2 \mathrm{H}), 7.25(\mathrm{t}, J=7.8 \mathrm{~Hz}, 1 \mathrm{H}), 6.73(\mathrm{~d}, J=16.2 \mathrm{~Hz}, 1 \mathrm{H}), 2.64(\mathrm{t}, J=7.4 \mathrm{~Hz}, 2 \mathrm{H}), 1.72$ (sext. app., $J=7.3 \mathrm{~Hz}, 2 \mathrm{H}), 0.99(\mathrm{t}, J=7.5 \mathrm{~Hz}, 3 \mathrm{H}) .{ }^{13} \mathrm{C} \mathrm{NMR}\left(\mathrm{CDCl}_{3}, 75 \mathrm{MHz}\right) \delta=200.0,140.4,136.8,133.1,130.8,130.4,127.3$, 126.8, 123.8, 43.1, 17.7, 13.8. MS (70 eV): m/z $(\%)=254(8), 252(8)[\mathrm{M}]^{+\bullet}, 211(85), 209(100)\left[\mathrm{M}-\mathrm{C}_{3} \mathrm{H}_{7}\right]^{+}, 183(26), 181$ (29) $\left[\mathrm{M}-\mathrm{COC}_{3} \mathrm{H}_{7}\right]^{+}, 173(65)[\mathrm{M}-\mathrm{Br}]^{+}, 102(63)\left[\mathrm{C}_{6} \mathrm{H}_{4} \mathrm{CHCH}\right]^{+\bullet}$. HRMS for $\mathrm{C}_{12} \mathrm{H}_{14} \mathrm{BrO}$ calculated 253.0223 and 255.0202 ; found 253.0224 and $255.0203[\mathrm{M}+\mathrm{H}]^{+}$.

1-(2-Methylphenyl)-hex-1-en-3-one 3n (entry 13):

\footnotetext{
${ }^{1}$ a) Darses, S.; Genet, J.-P. Eur. J. Org. Chem. 2003, 4313. (b) Darses, S.; Michaud, G.; Genet, J.-P. Eur. J. Org. Chem. $1999,1875$.
} 
Reaction of potassium 3-methylphenyltrifluoroborate (1 mmol, $198 \mathrm{mg})$ and hex-1-en-3-one $(2 \mathrm{mmol}, 48 \mathrm{mg})$ afforded $162 \mathrm{mg}$ of enone $3 \mathrm{n}$ as a pale yellow oil (yield $86 \%)$. GC $\left(50 \mathrm{kPa}, 70^{\circ} \mathrm{C}\right.$ during 1 min then $20^{\circ} \mathrm{C} / \mathrm{min} \mathrm{up}$ to $\left.210^{\circ} \mathrm{C}\right)$ : $\mathrm{t}_{\mathrm{R}} 8.20$ min. Rf (petroleum ether / ethyl acetate 4/1): 0.78. ${ }^{1} \mathrm{H} \mathrm{NMR}\left(\mathrm{CDCl}_{3}, 300 \mathrm{MHz}\right) \delta=7.86(\mathrm{~d}, J=16.0 \mathrm{~Hz}, 1 \mathrm{H}), 7.56-7.59(\mathrm{~m}$, $1 \mathrm{H}), 7.11-7.31(\mathrm{~m}, 3 \mathrm{H}), 6.67(\mathrm{~d}, J=16.0 \mathrm{~Hz}, 1 \mathrm{H}), 2.65(\mathrm{t}, J=7.3 \mathrm{~Hz}, 2 \mathrm{H}), 2.45(\mathrm{~s}, 3 \mathrm{H}), 1.73(\mathrm{sext} . \mathrm{app} ., J=7.4 \mathrm{~Hz}, 2 \mathrm{H})$, $0.99(\mathrm{t}, J=7.4 \mathrm{~Hz}, 3 \mathrm{H}) \cdot{ }^{13} \mathrm{C} \mathrm{NMR}\left(\mathrm{CDCl}_{3}, 75 \mathrm{MHz}\right) \delta=200.4,139.8,137.9,133.6,130.8,130.1,127.2,126.4,126.3,43.1$,

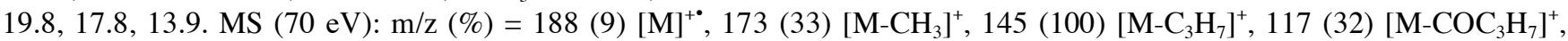
115 (51), 91 (23) $\left[\mathrm{CH}_{3} \mathrm{C}_{6} \mathrm{H}_{4}\right]^{+}$. HRMS for $\mathrm{C}_{13} \mathrm{H}_{17} \mathrm{O}$ calculated 189.1274; found $189.1273[\mathrm{M}+\mathrm{H}]^{+}$.

All other products were identified by comparison of spectroscopic data with authentic sample or with those reported in the literature: 1b [83160-20-7], 3a [52392-64-0], 3b [125950-99-4], 3c [114833-06-6], 3d [346586-17-2], 3e [220466-27-3], 3f [352-03-4], 3g [24393-48-4], 3h [527759-23-5], 3i [202476-39-9], 3j [202476-40-2], 3k [99564-46-2], 31 [99564-62-2], 3o [103-36-6], 5a [116144-99-1], 5b [87482-94-8]. 

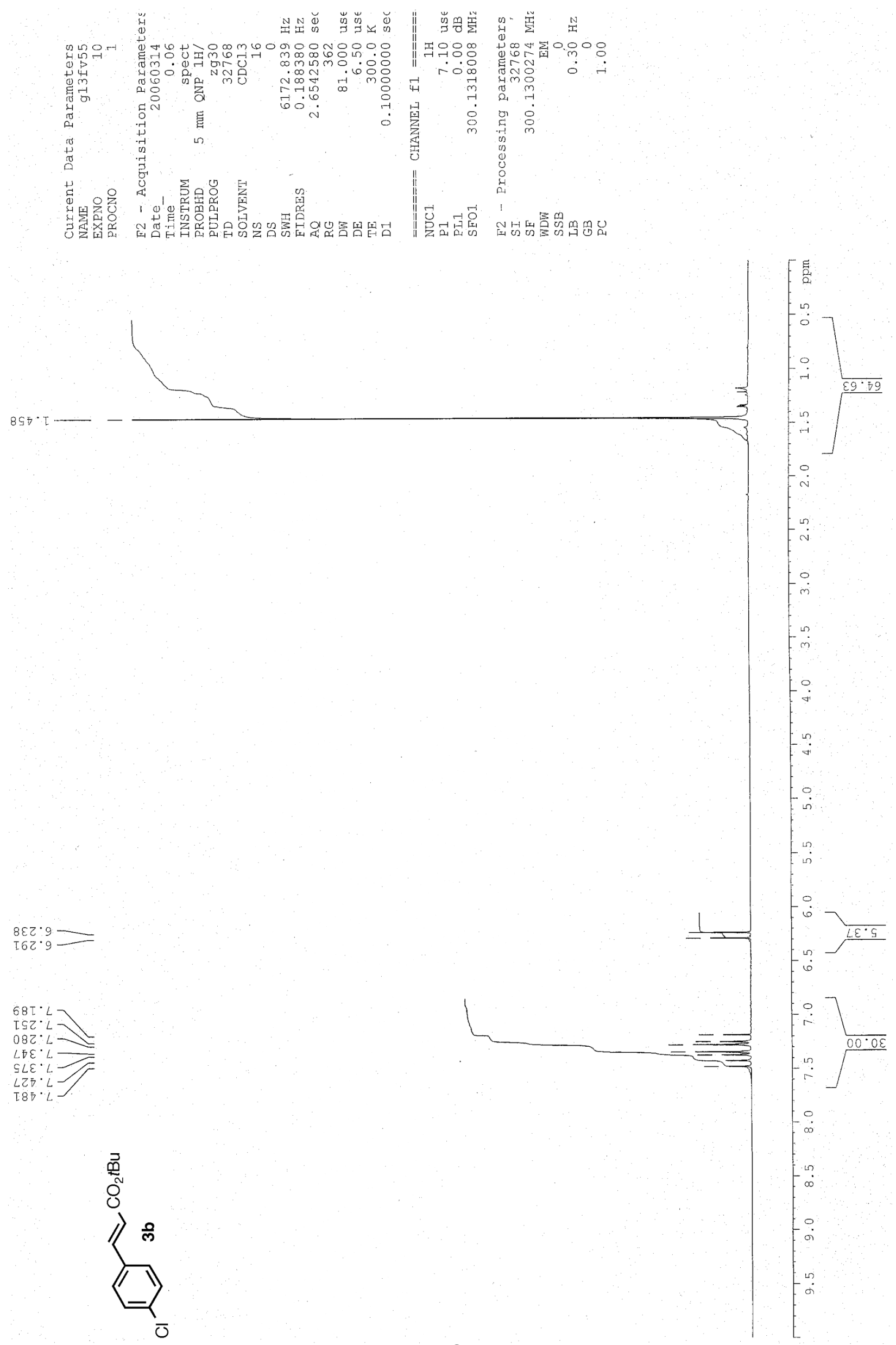

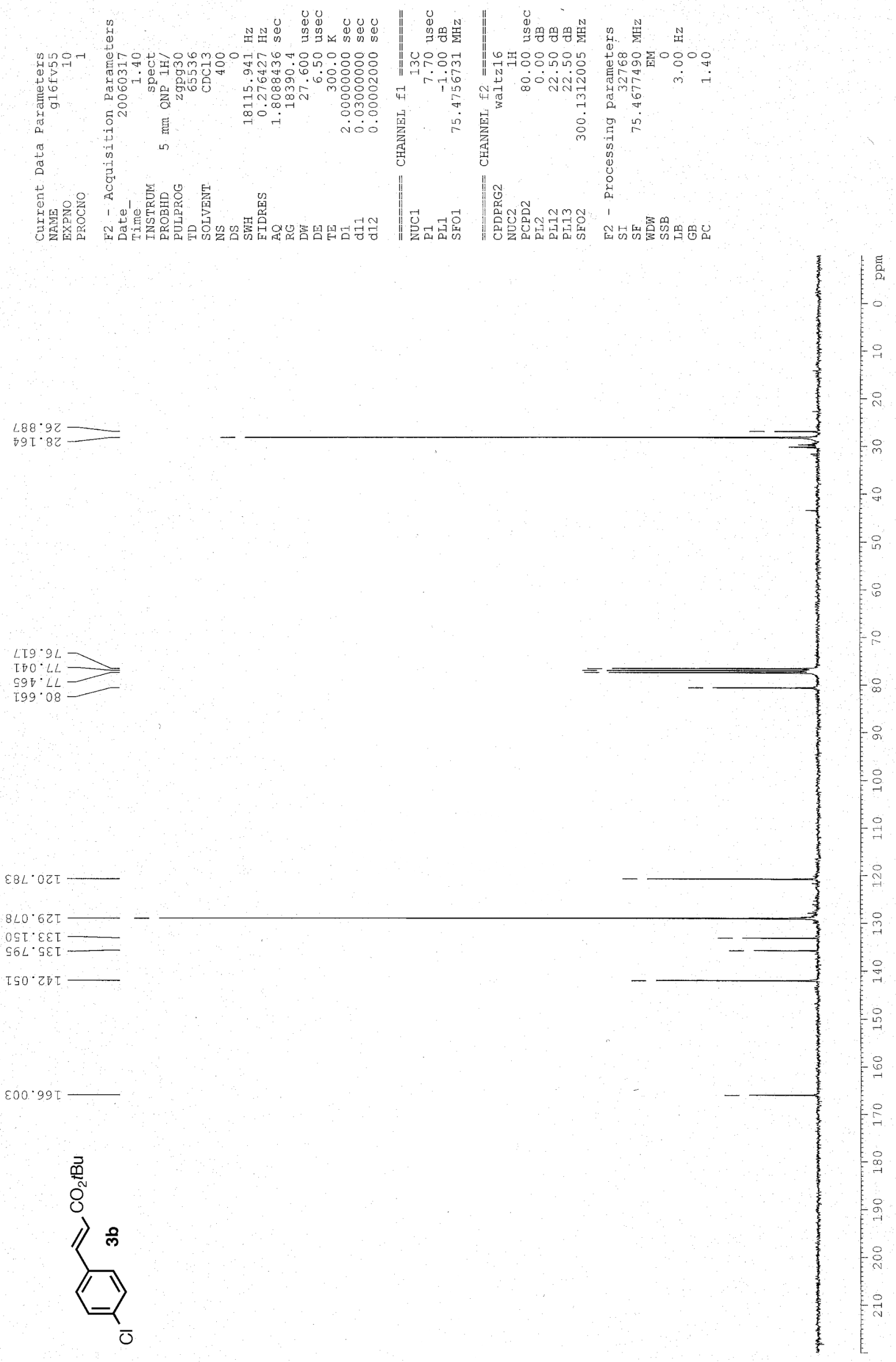

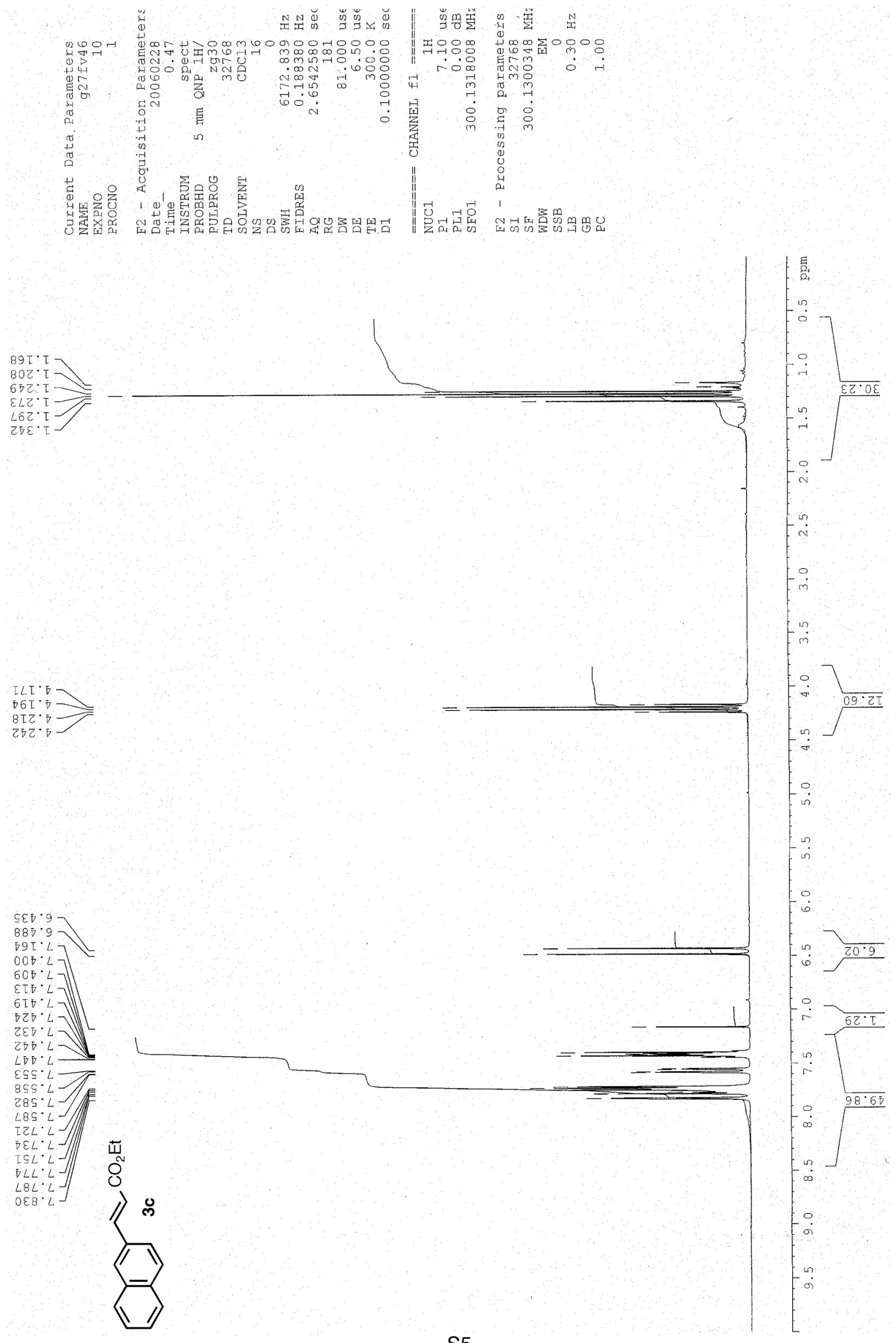
*

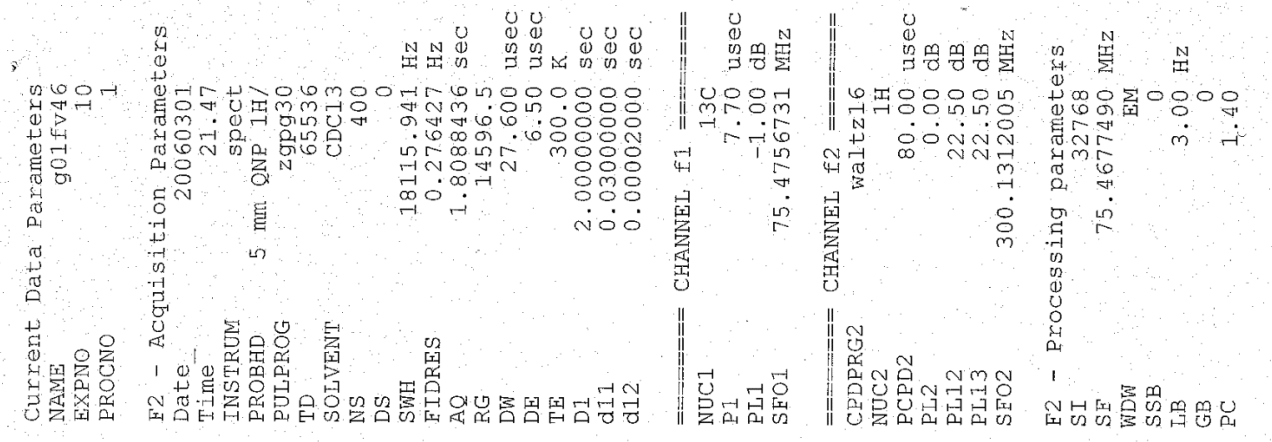

$08 \varepsilon^{\circ} \neq 1$

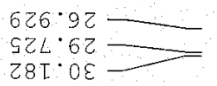

$825 \cdot 09$

$1 \angle 9^{\circ} 9 L-$
$\angle 60^{\circ} \angle L C$
$T Z G^{\circ} L L$

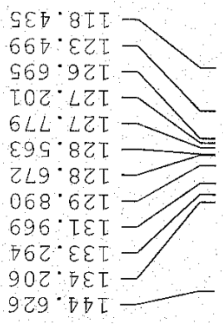

$590: \angle 9 I \longrightarrow$<smiles>O/C=C/c1ccc2ccccc2c1</smiles>

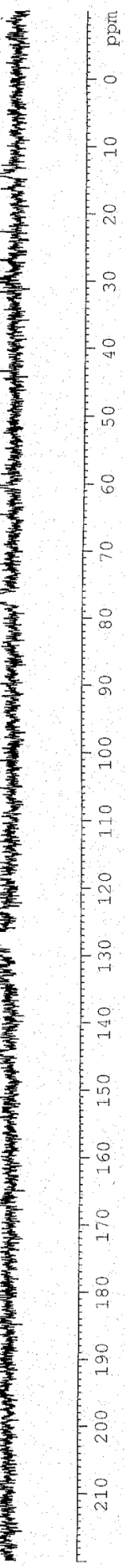

S6 

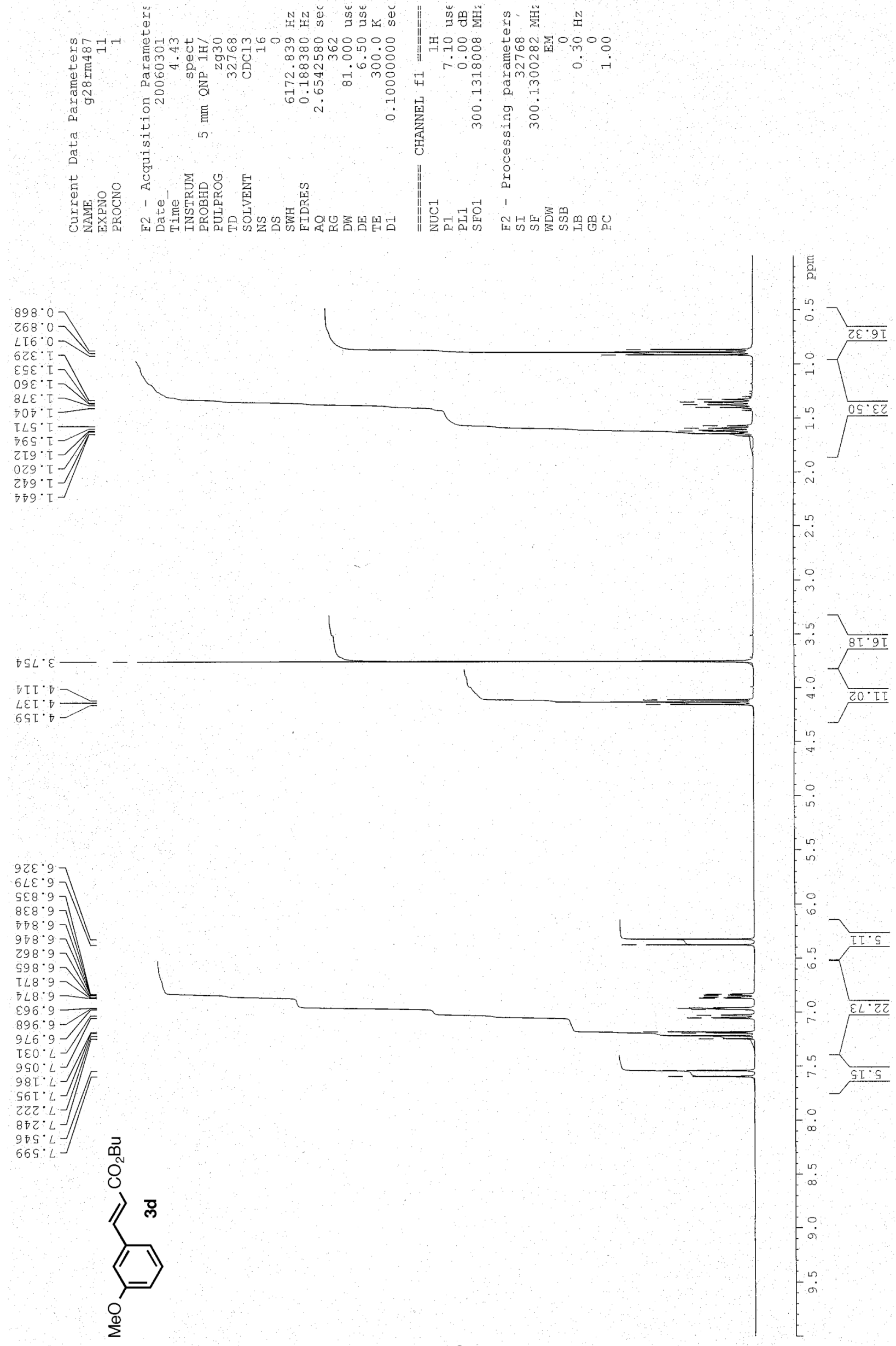

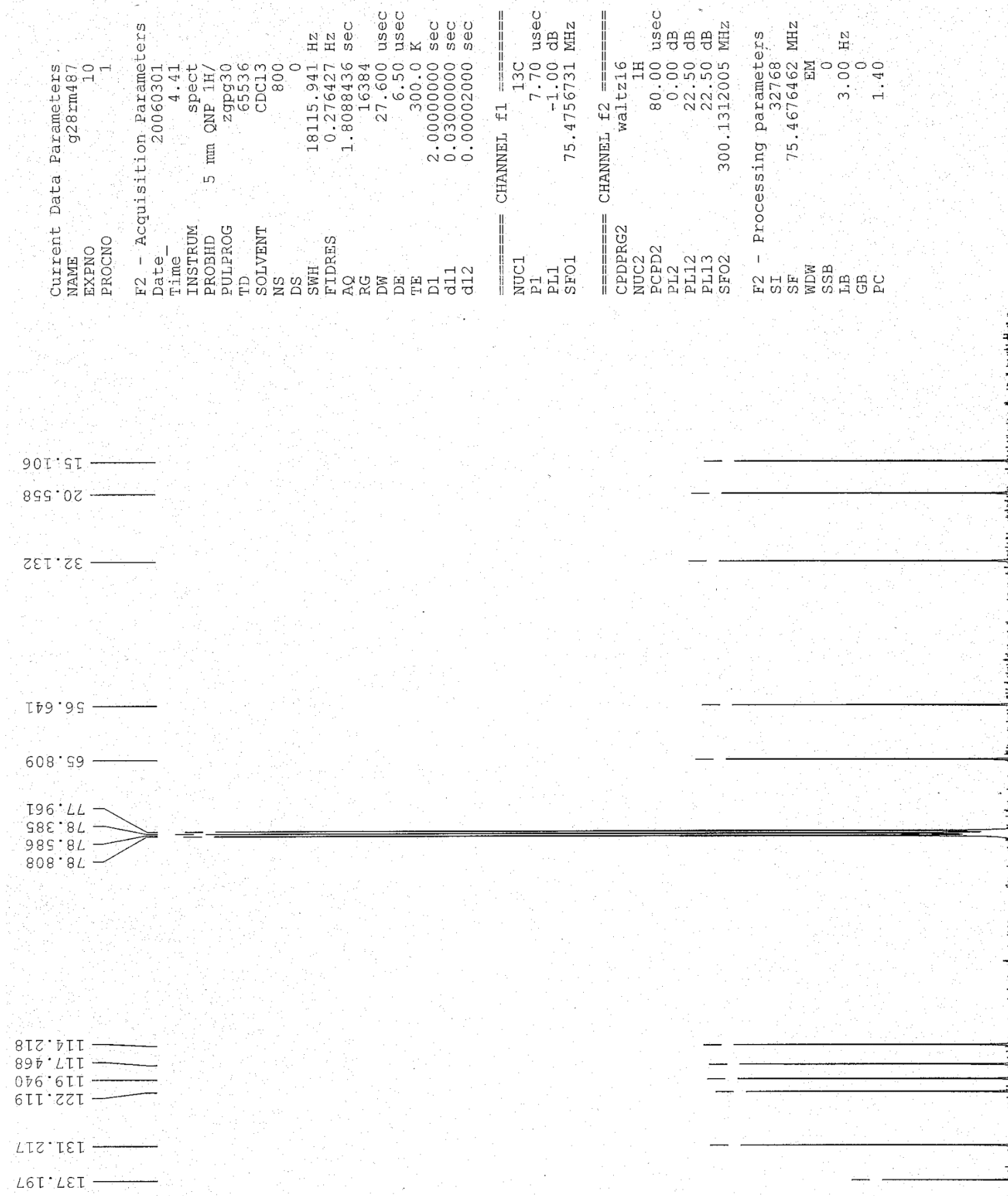

IZ8. SET

SषZT9T $\longrightarrow$

$\llcorner 6 \varepsilon \cdot 89 \tau$

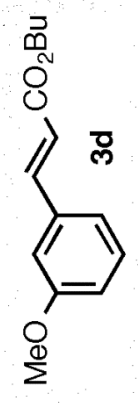



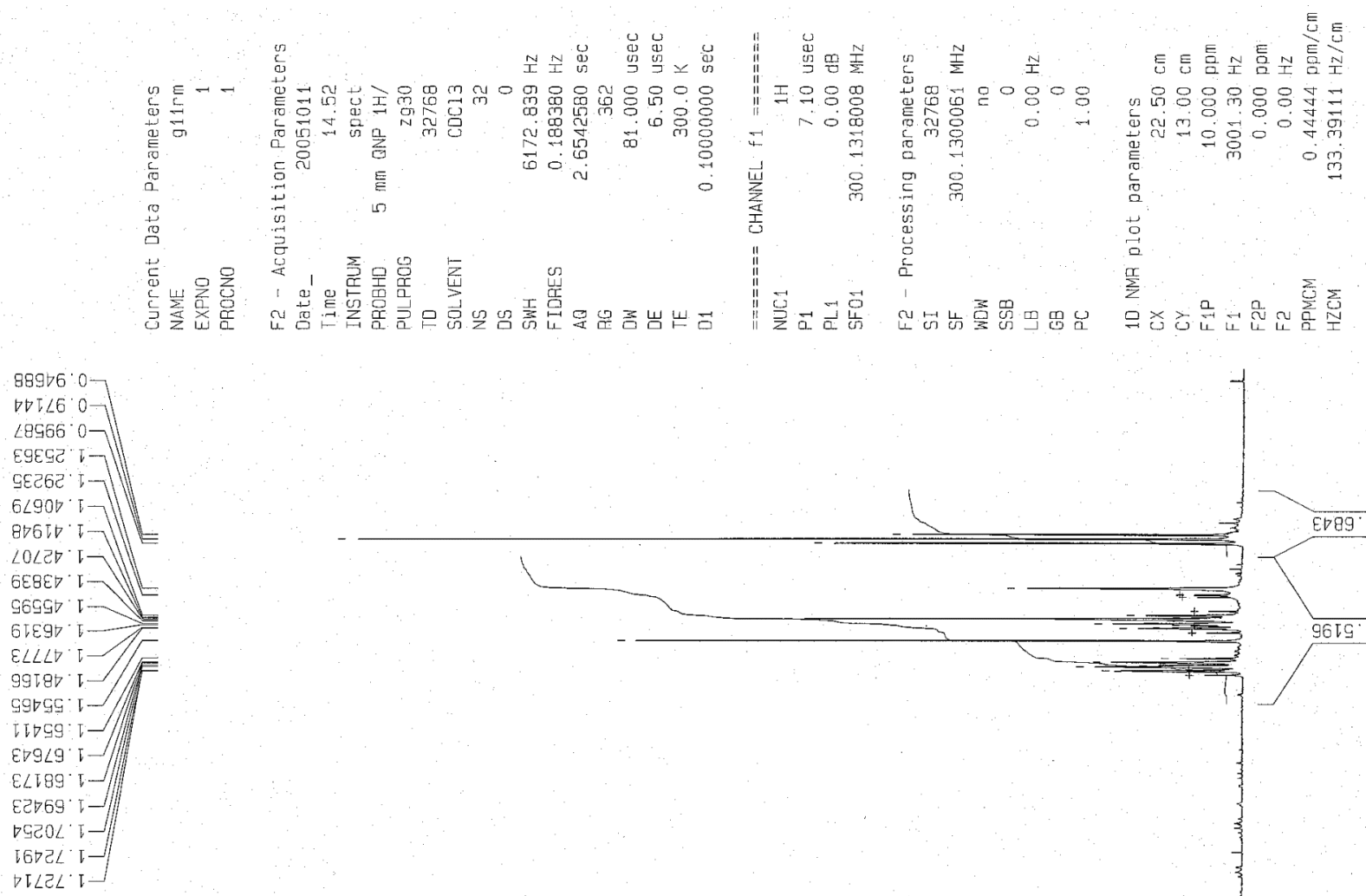

$50 \angle 0 e^{\circ} \nabla$

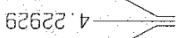

g力 เ

.6987:9-

$9 \nabla 6 E 9 \cdot 9$

$09092: \angle-$

$92 \nabla 09 \cdot \angle$

$08909^{\circ} \angle$

09829 $\angle=$

$29989^{\circ} \angle$

โ9799: $\angle$

$6 \nabla \angle 99: \angle-$

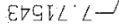

$\overline{0}$
$\overline{0}$
$\overline{0}$
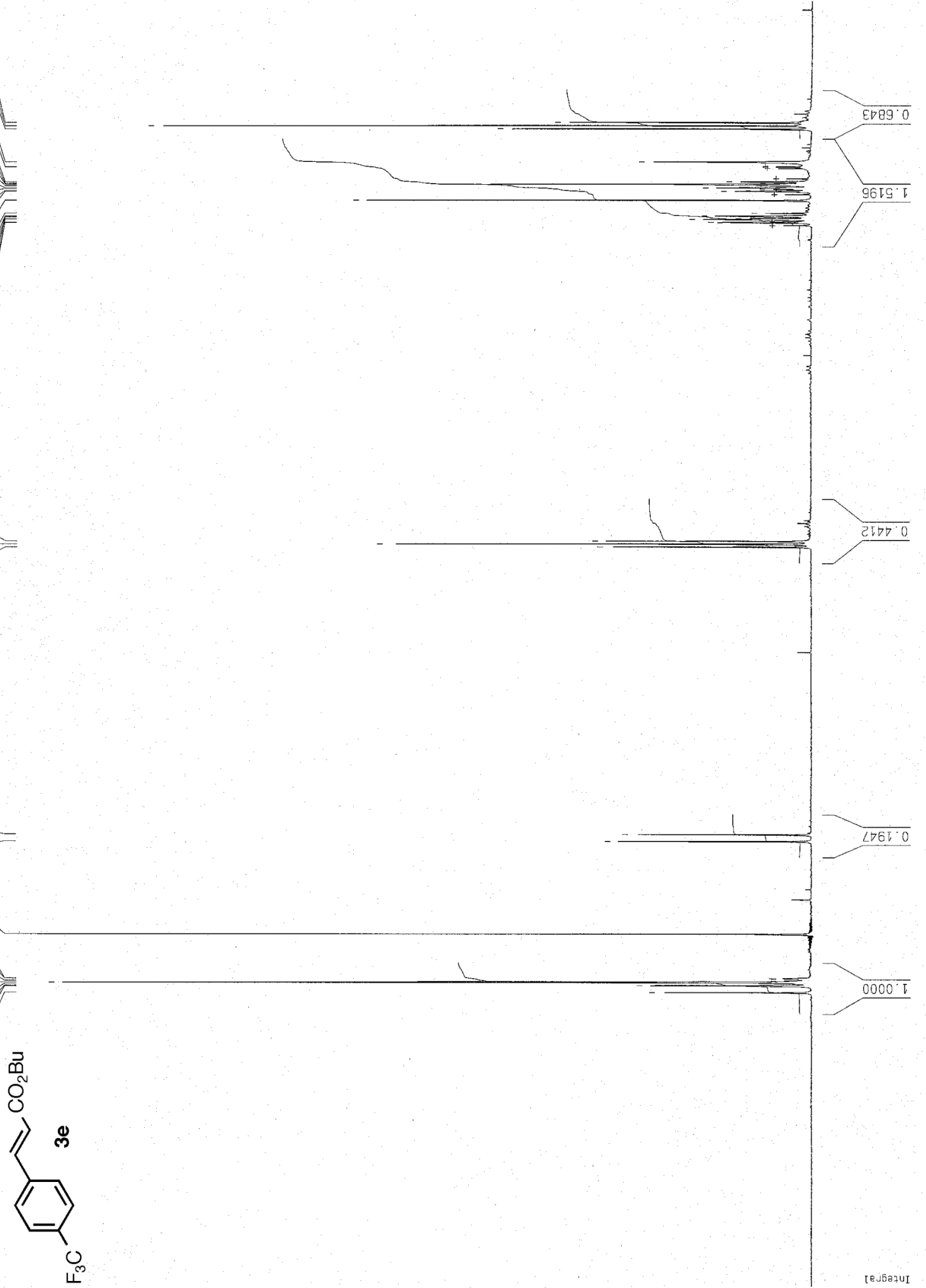

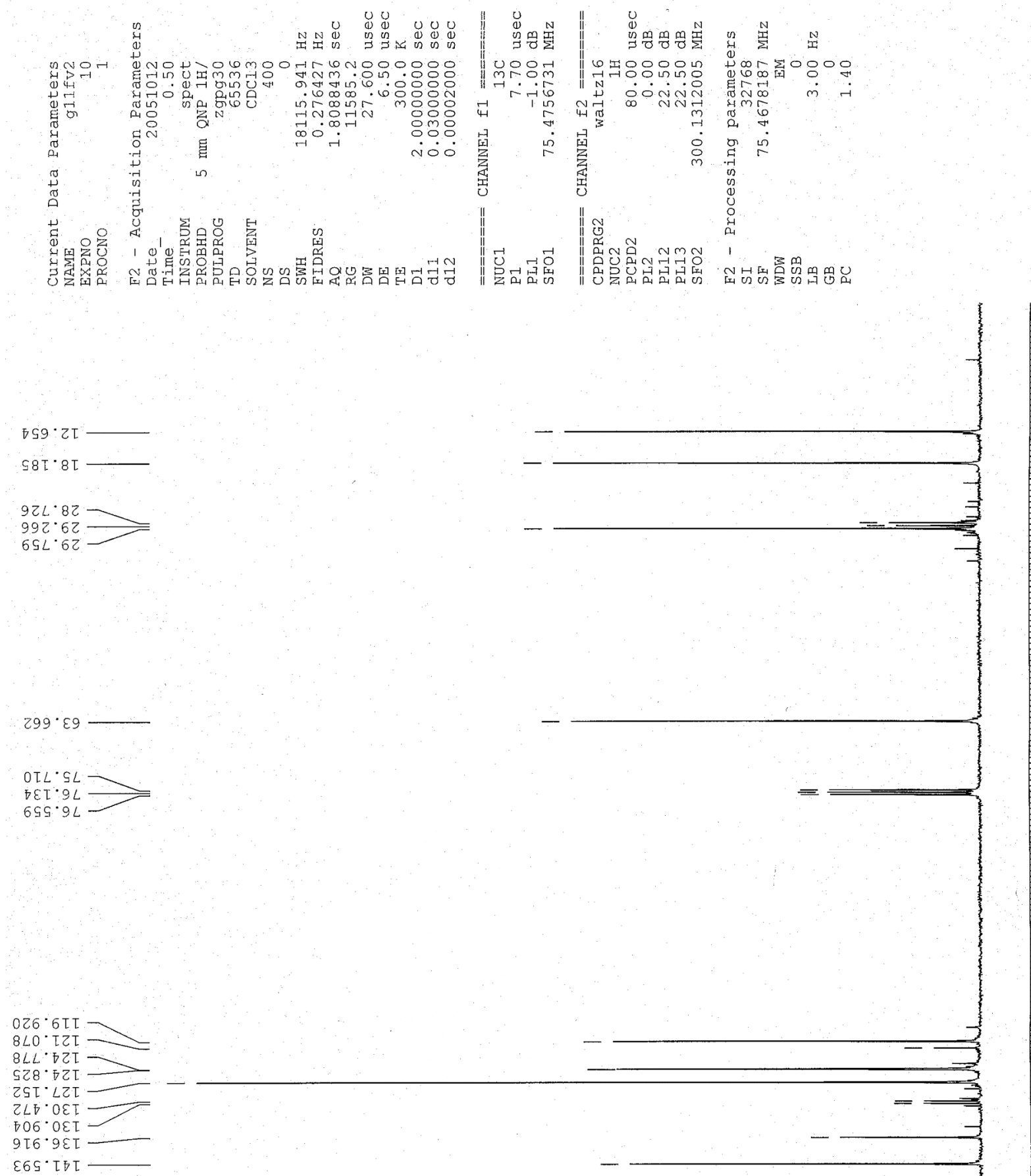

$\varepsilon 6 S^{\circ} T E T$

$607 \cdot 59 I$
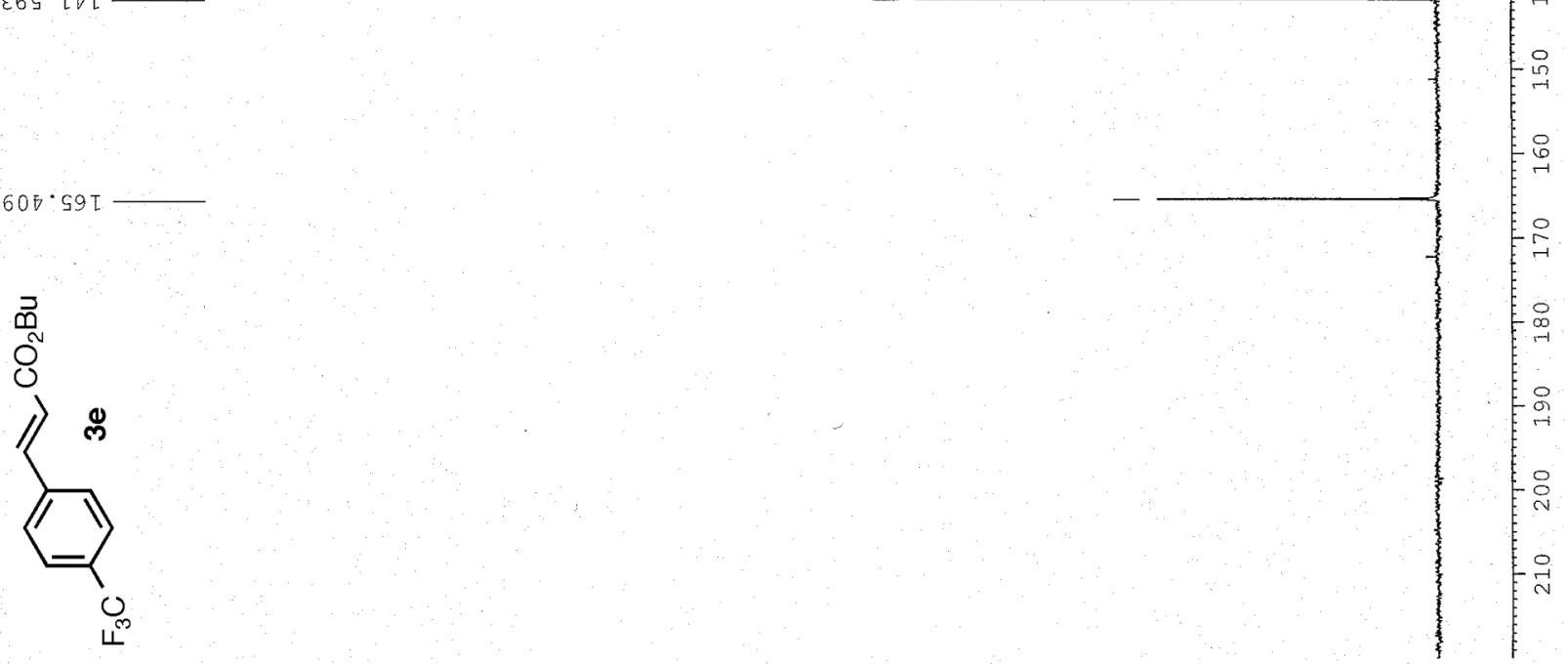

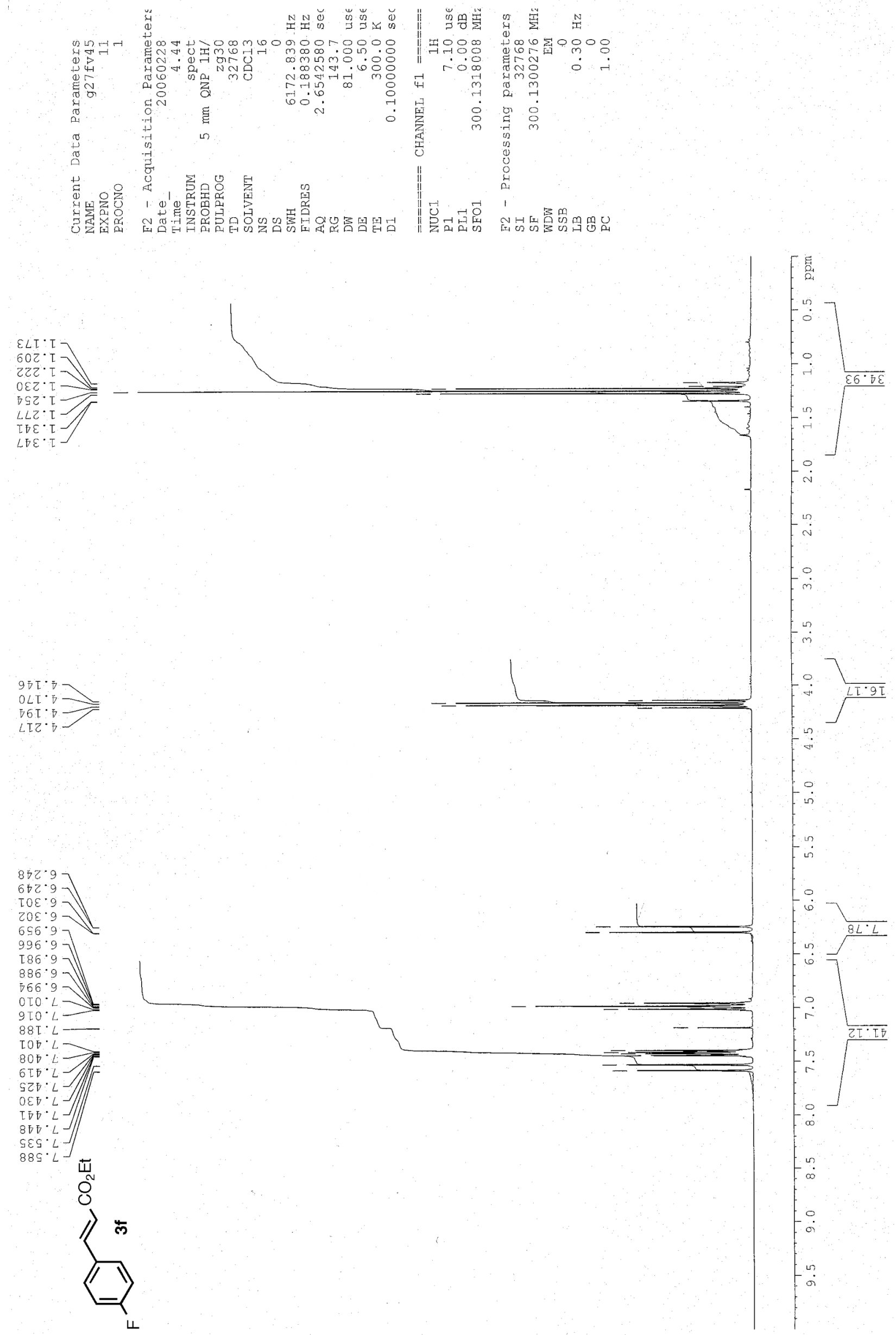

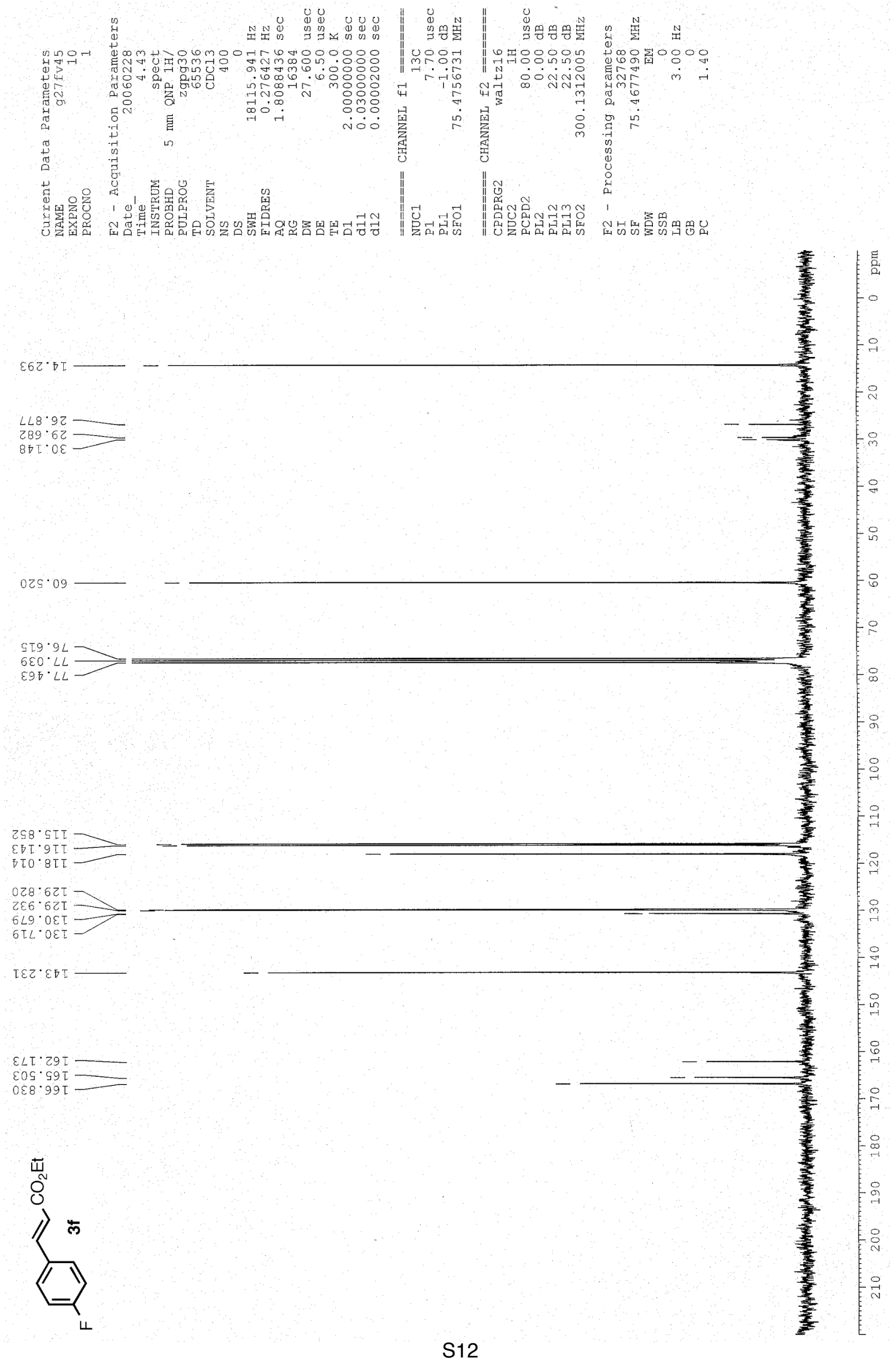

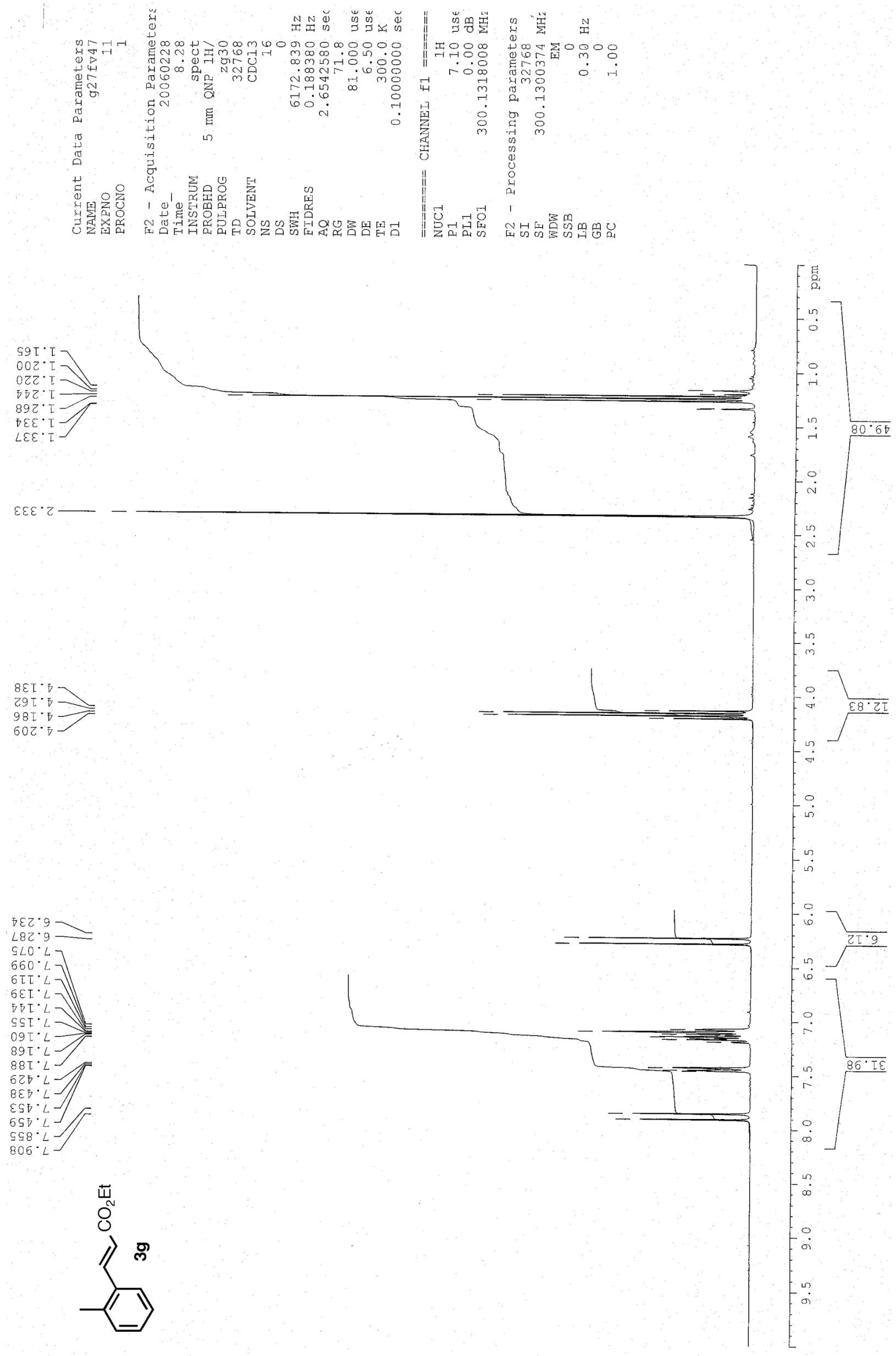

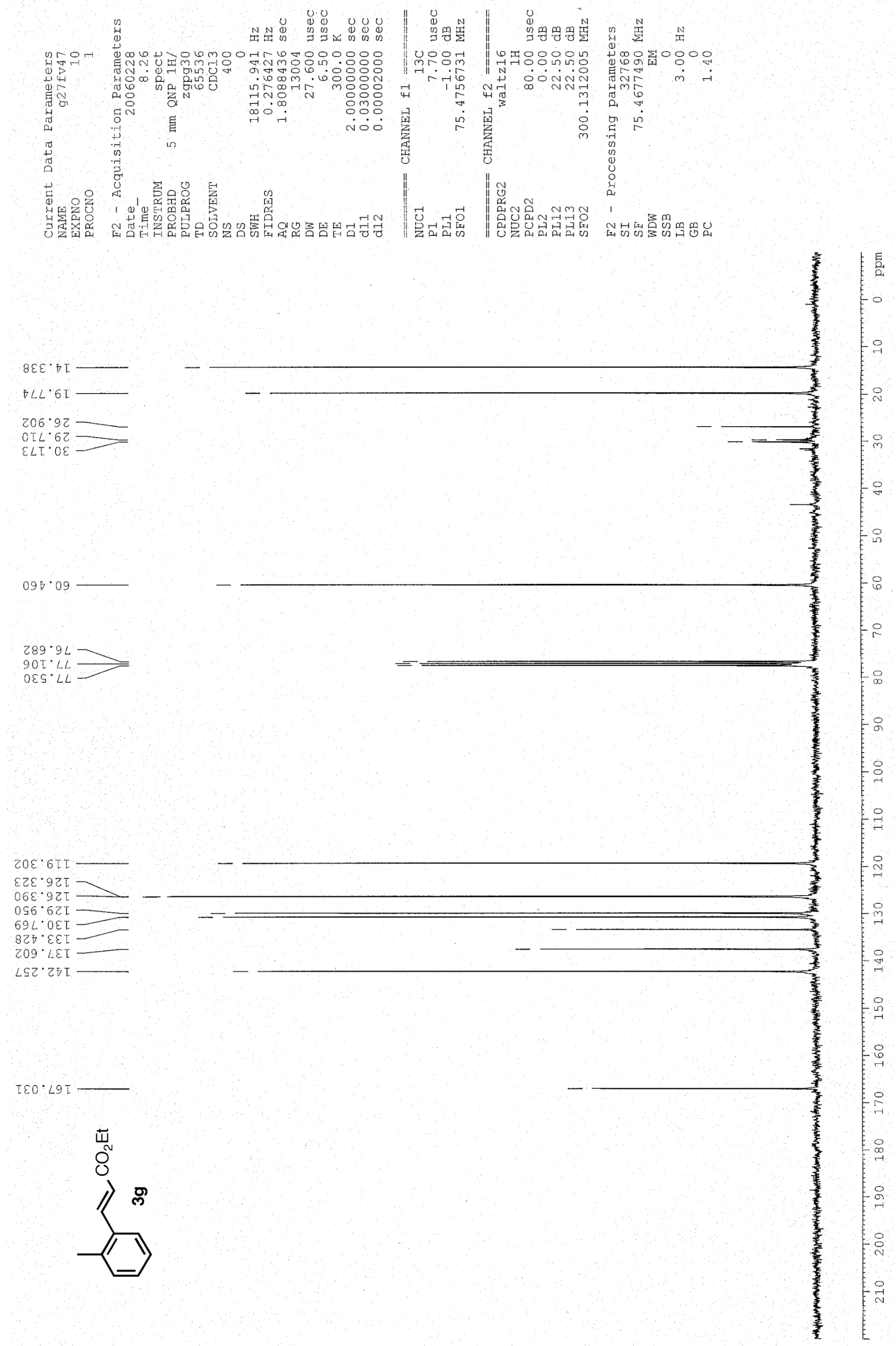

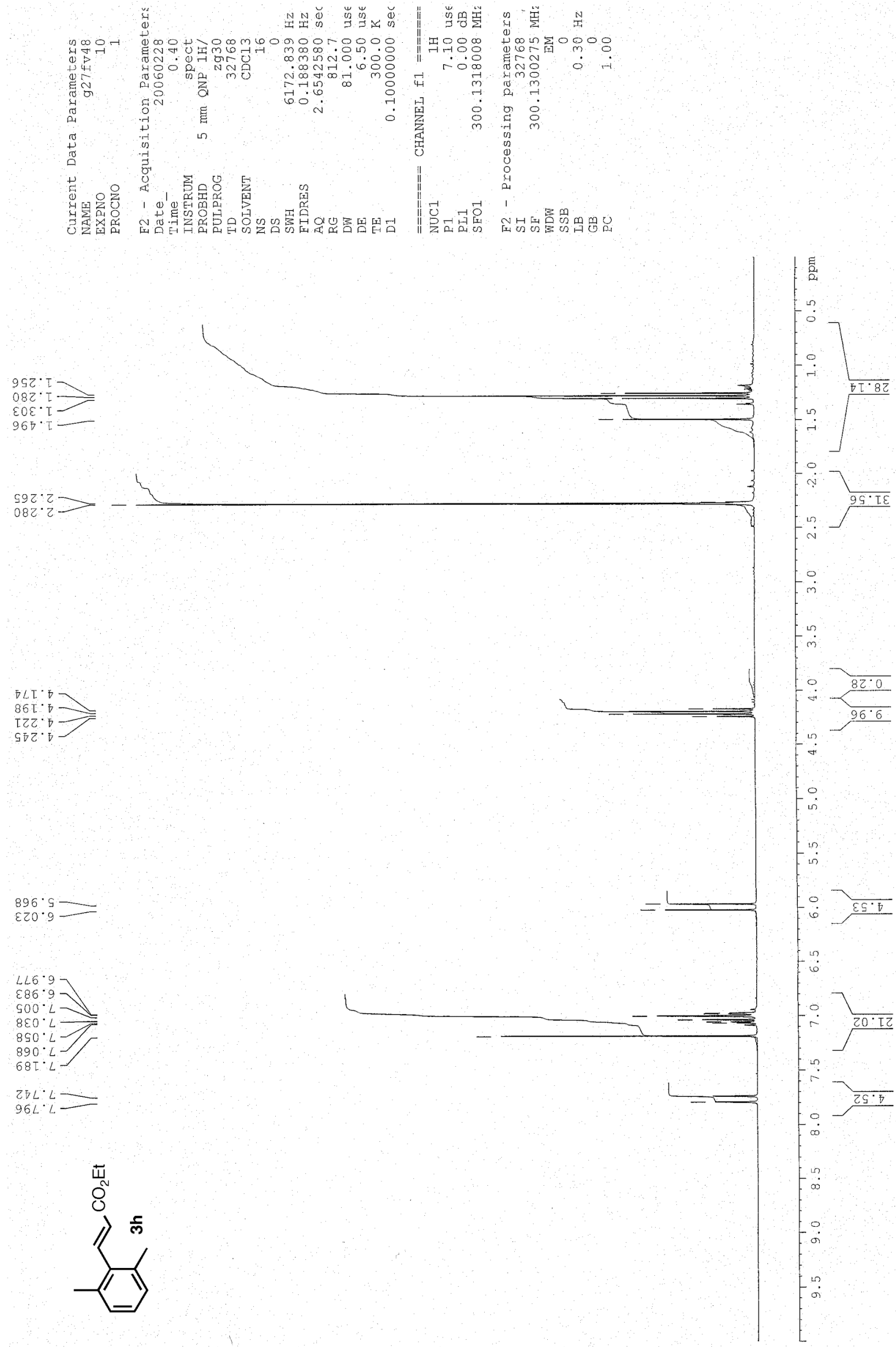

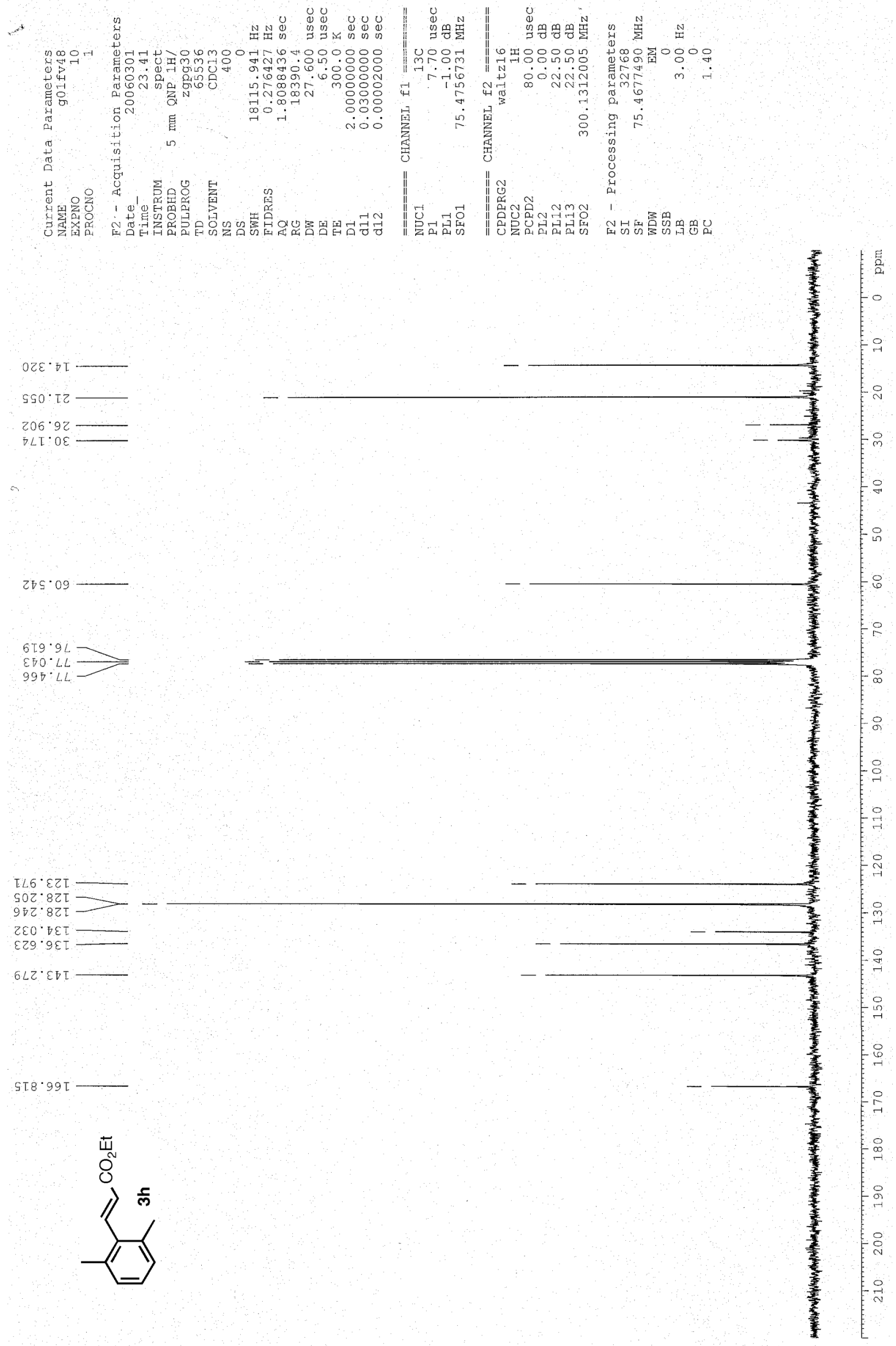

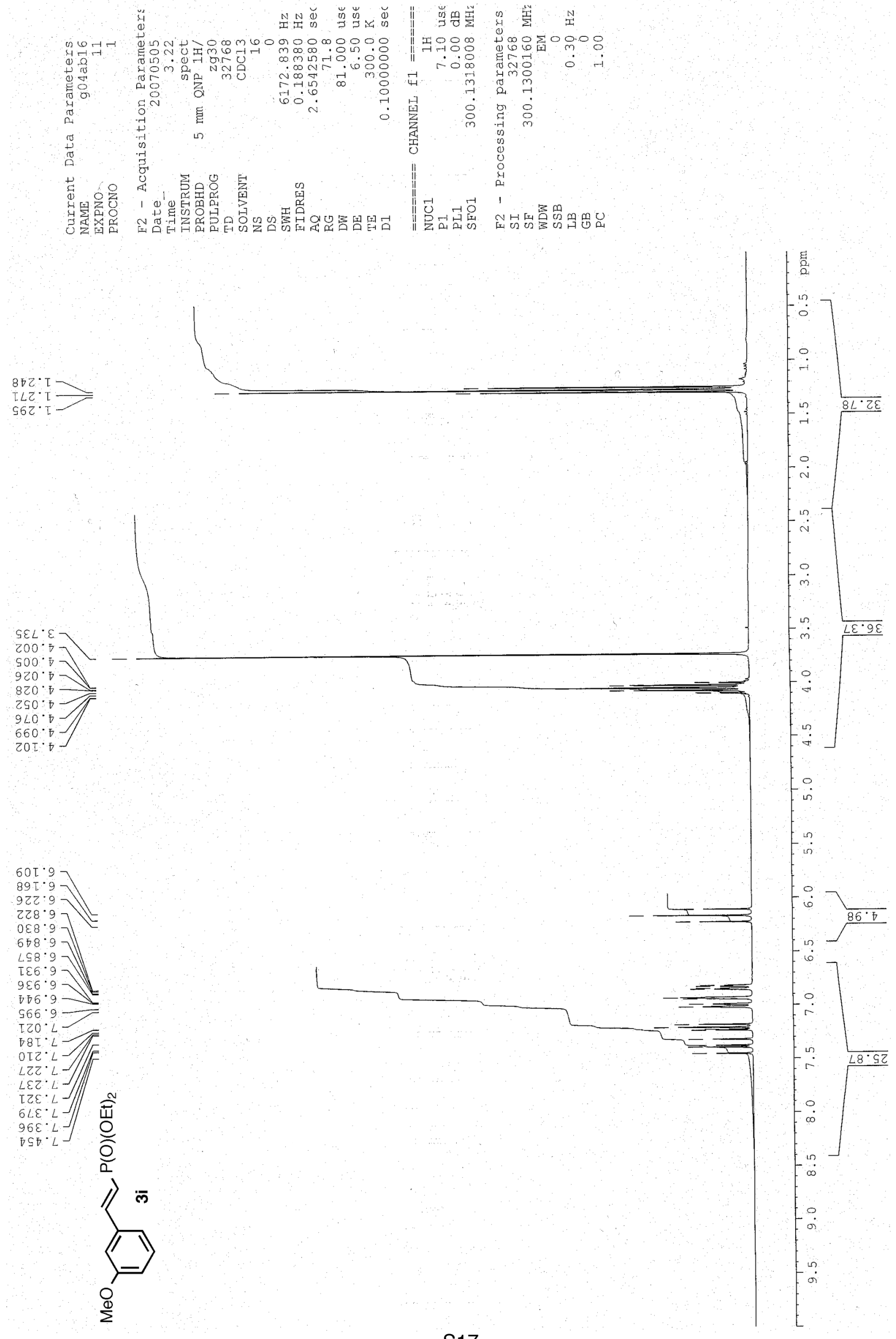

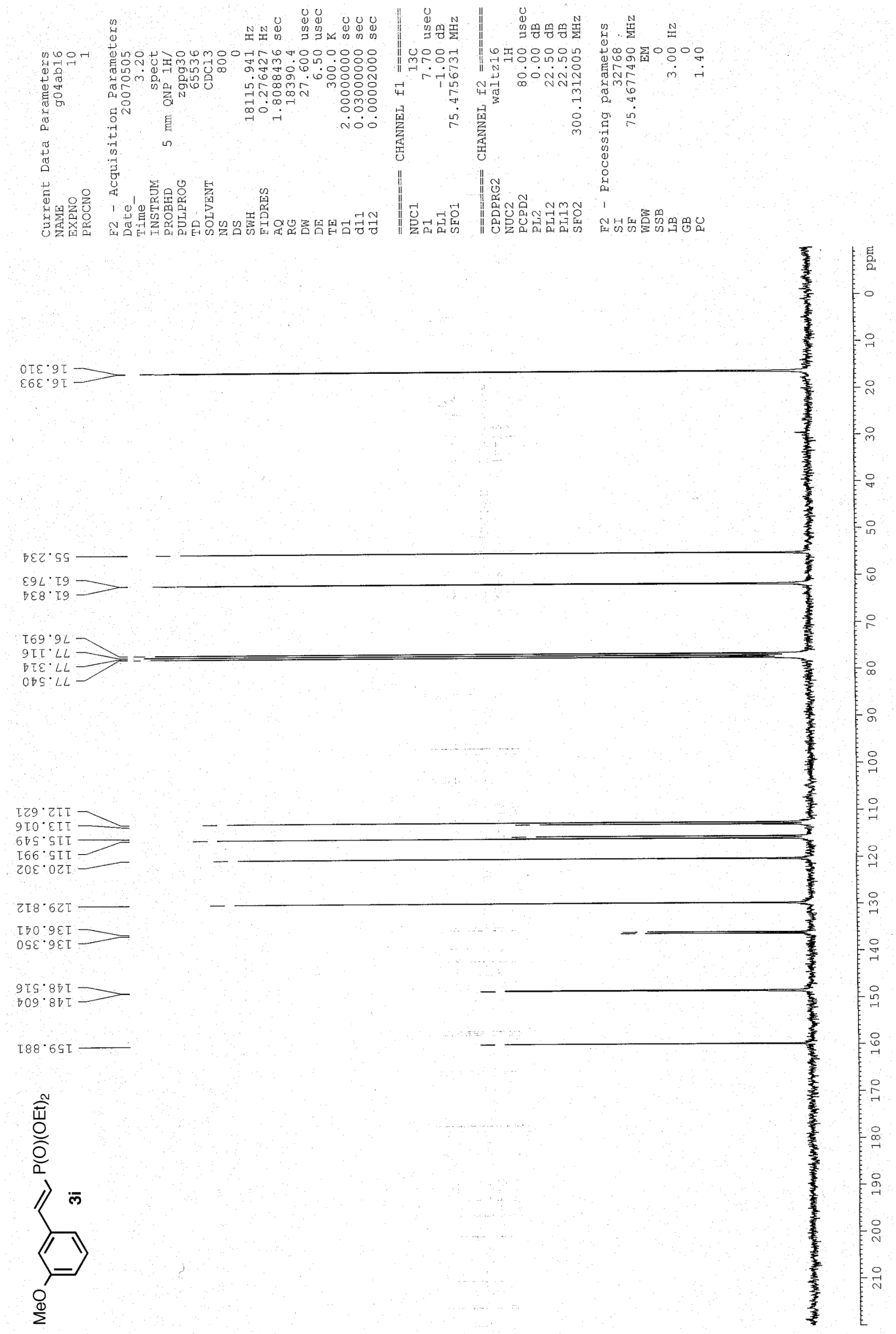

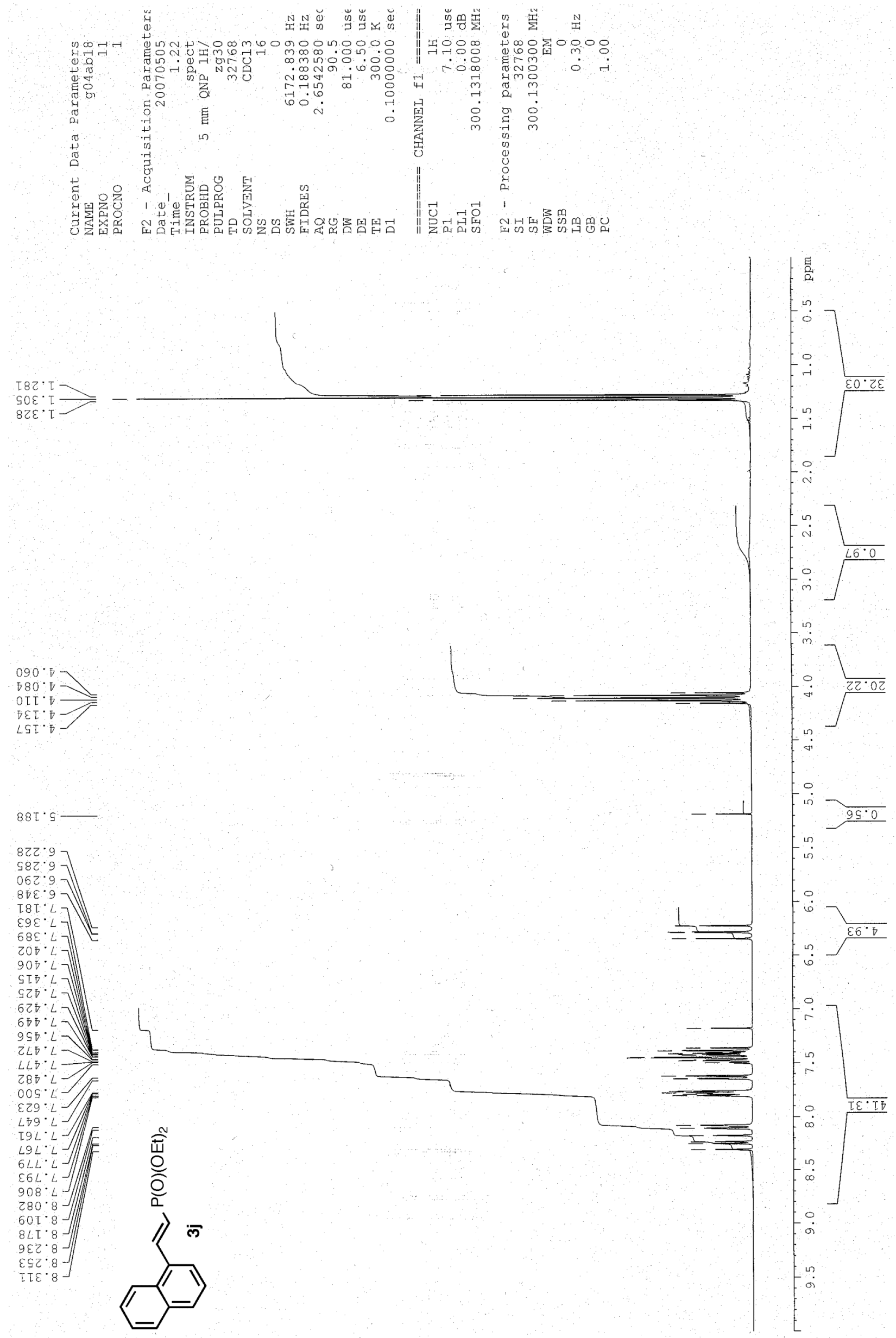

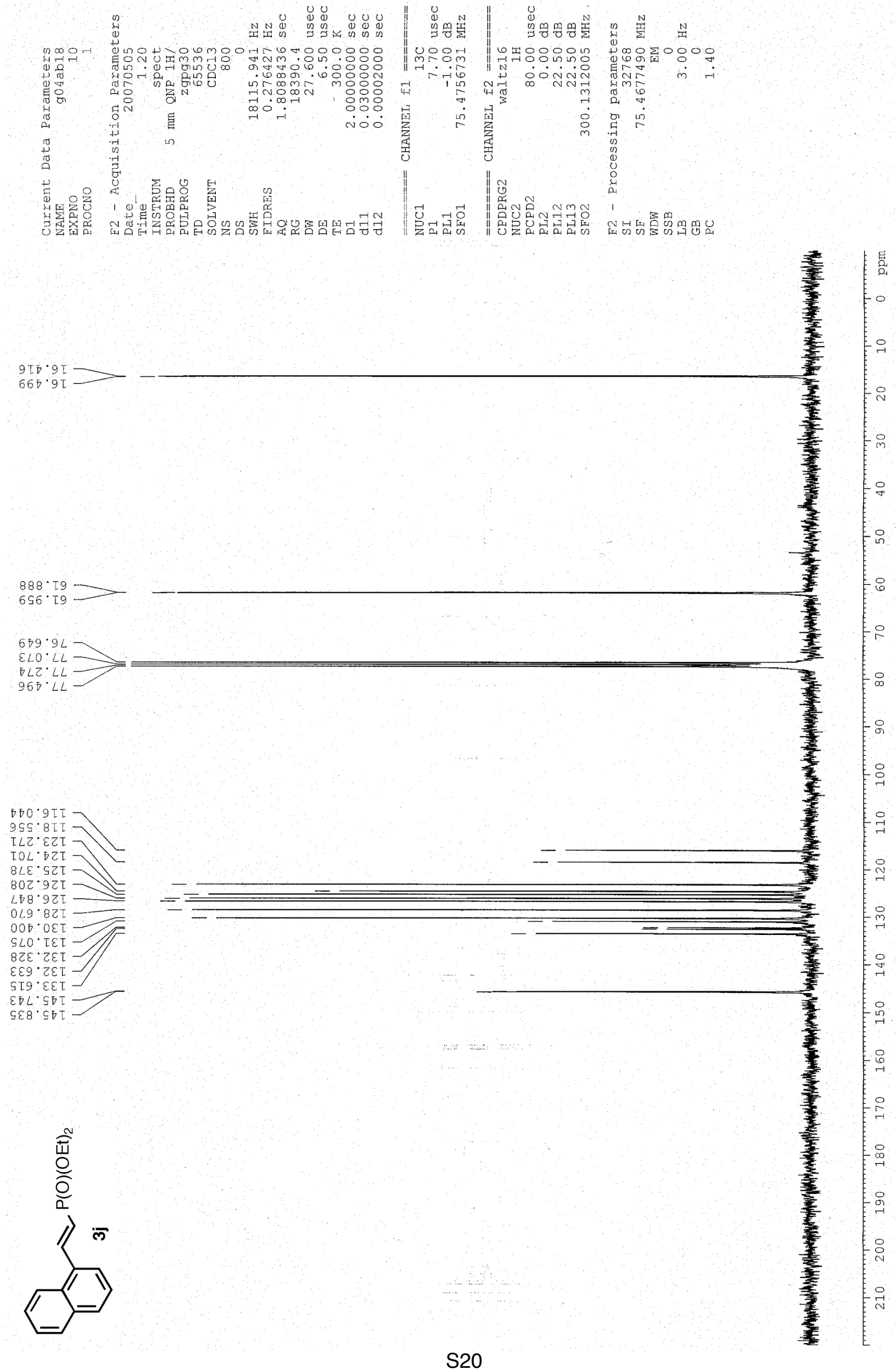

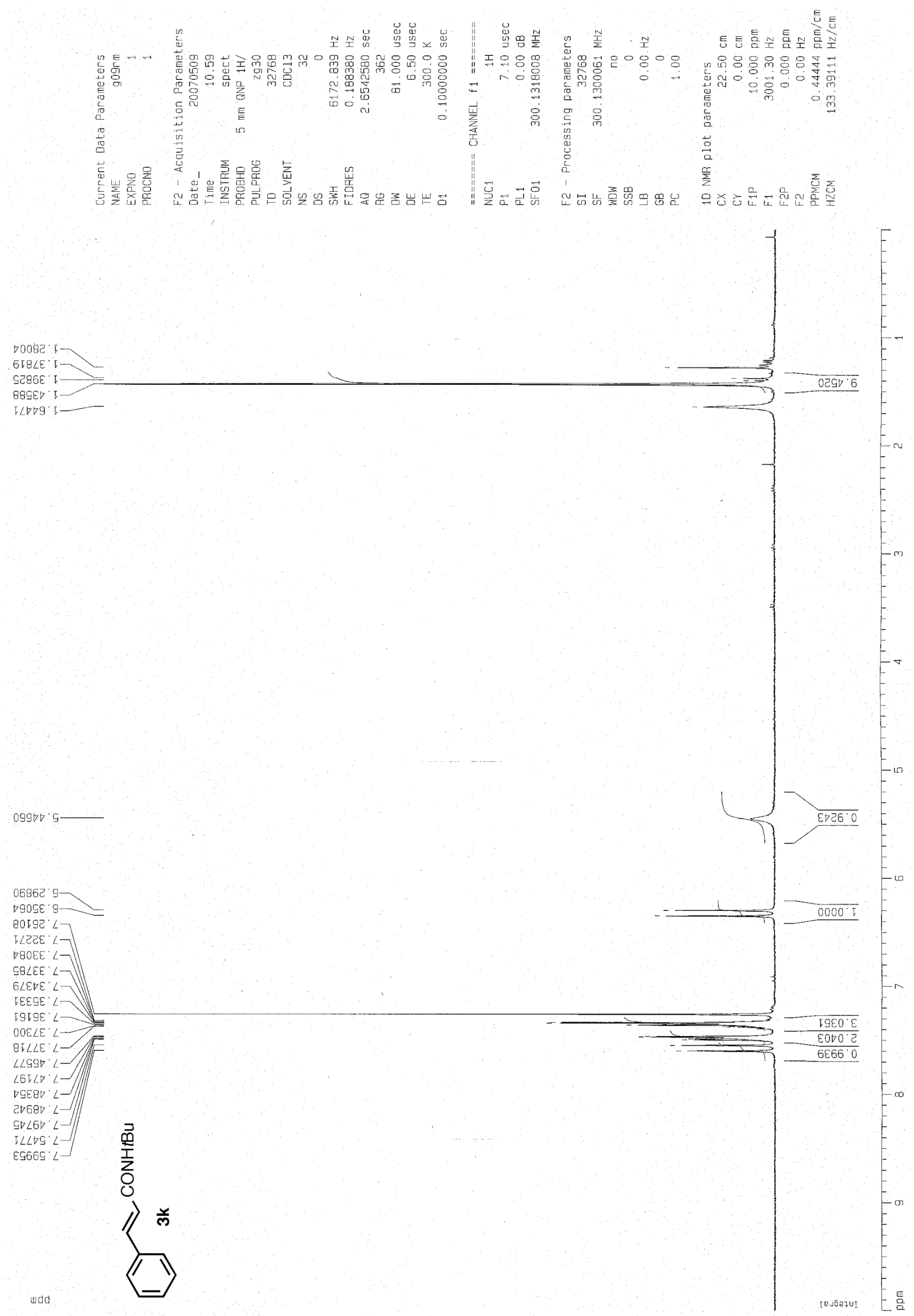


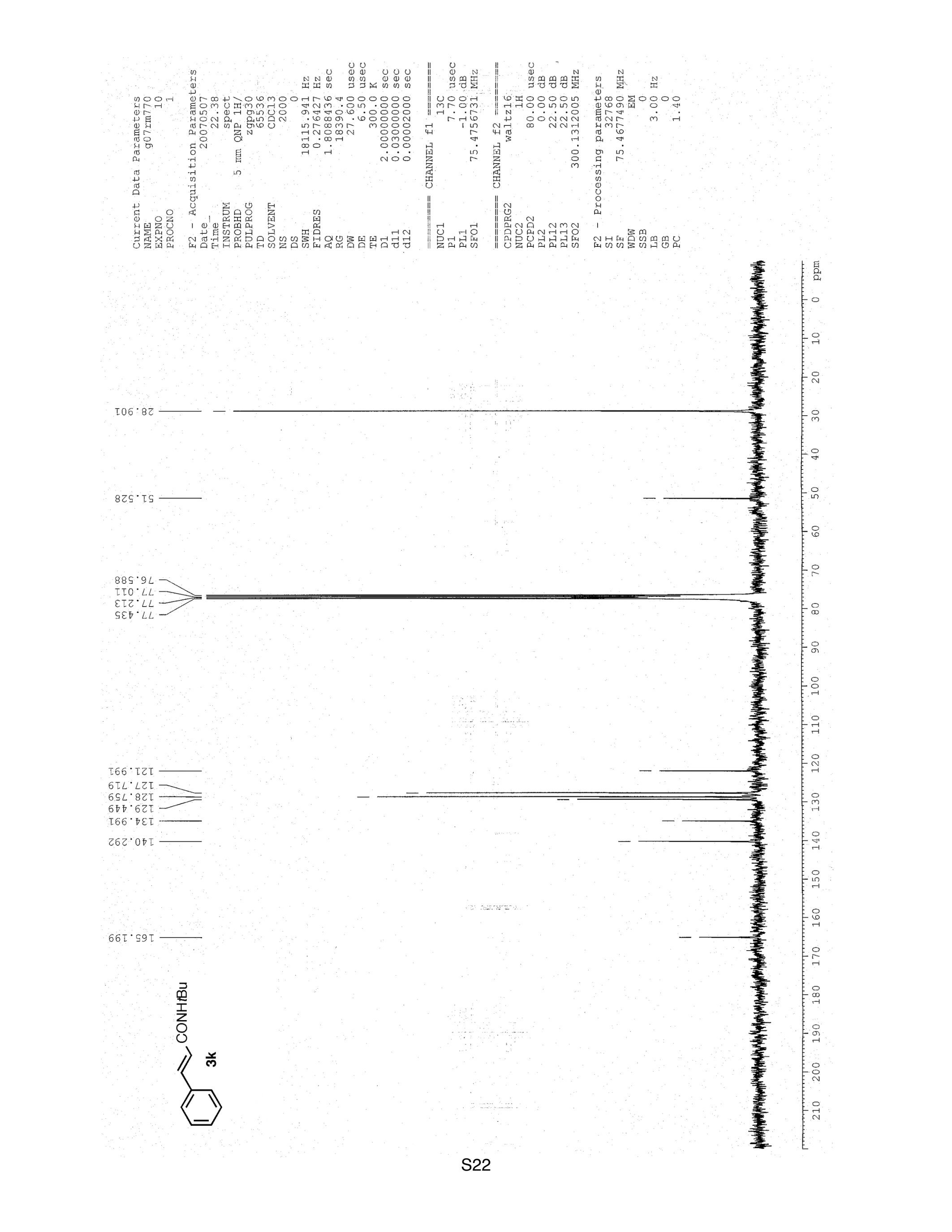



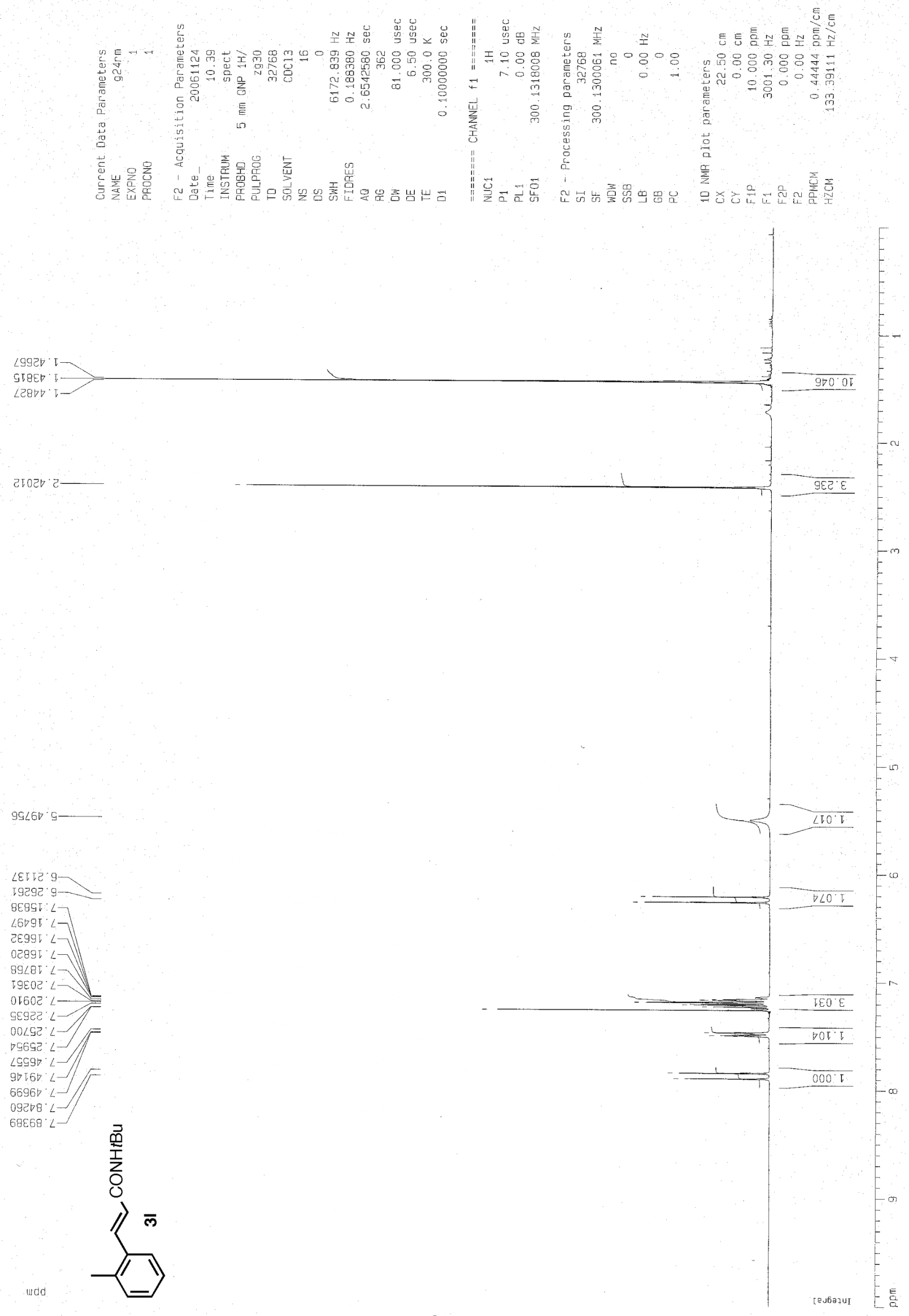

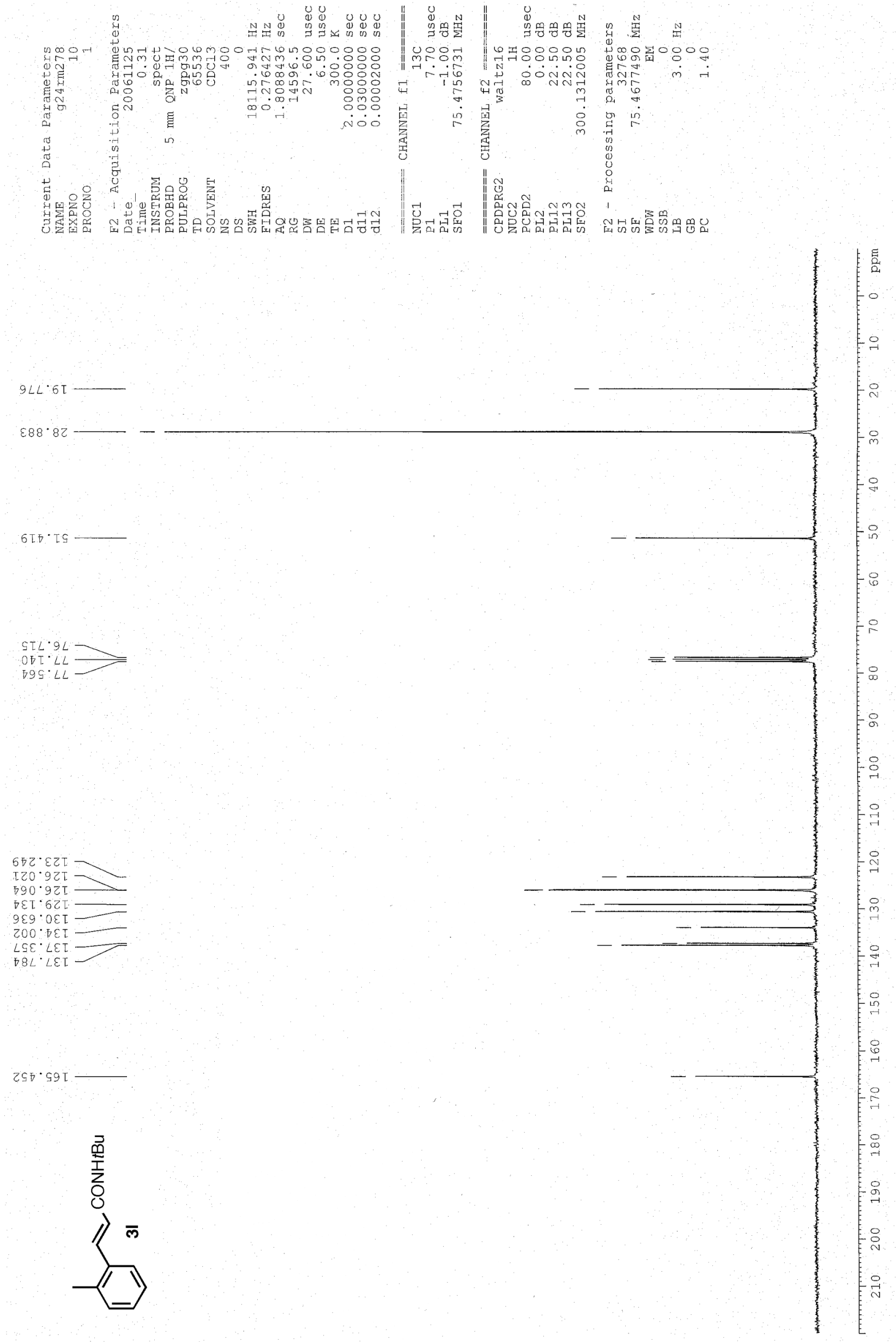

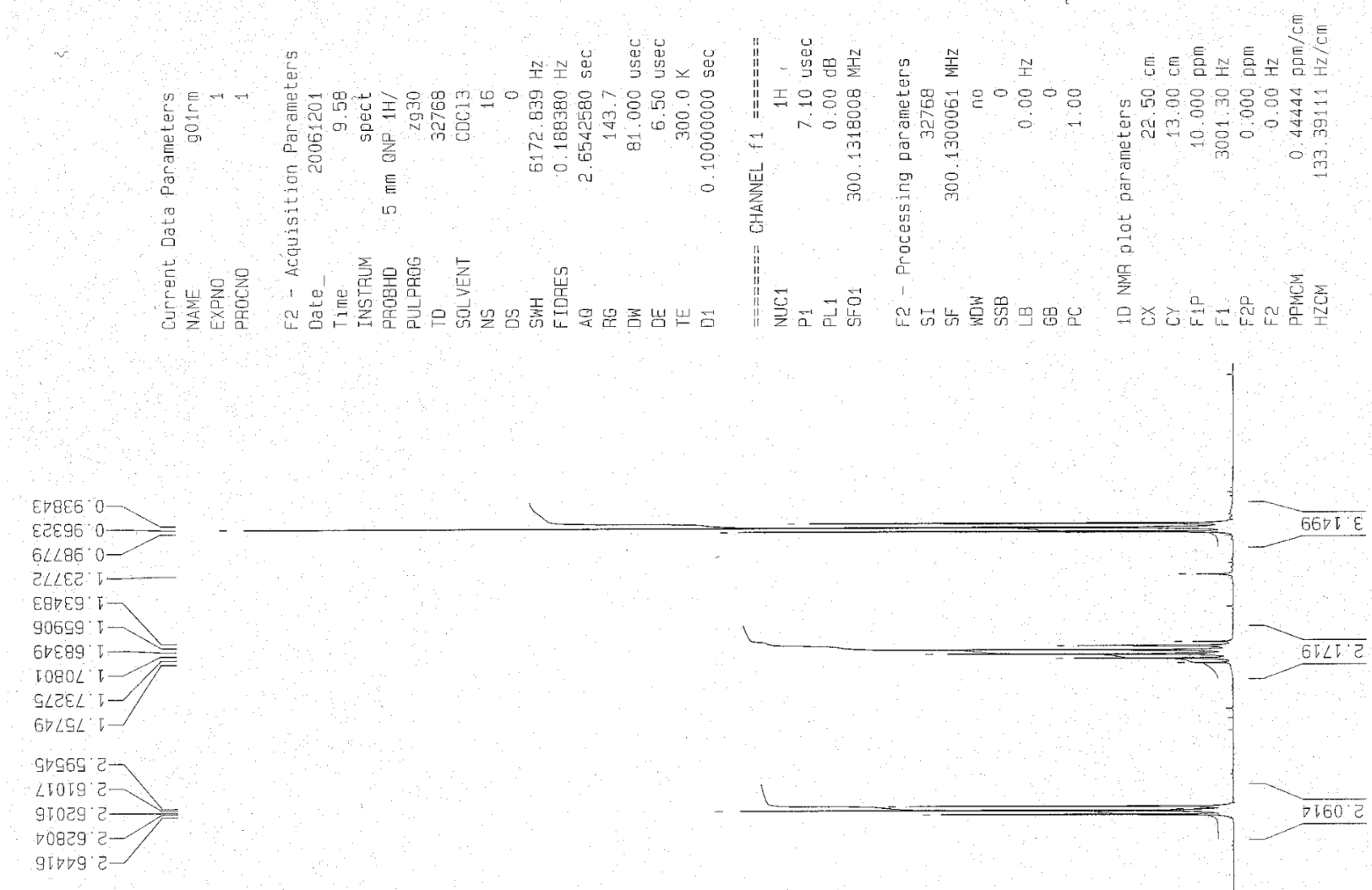

$5008 \mathrm{~g}-$

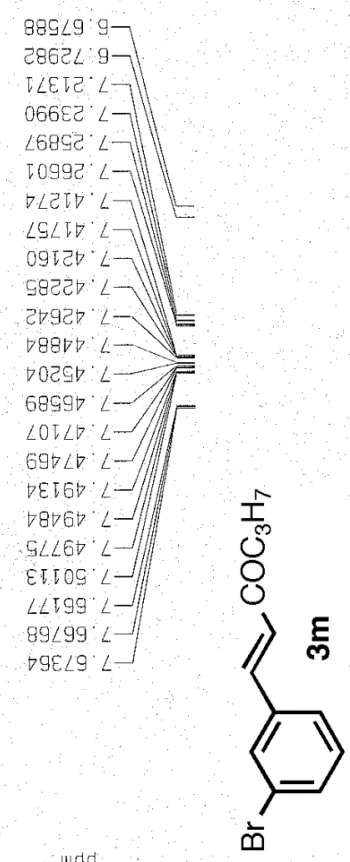



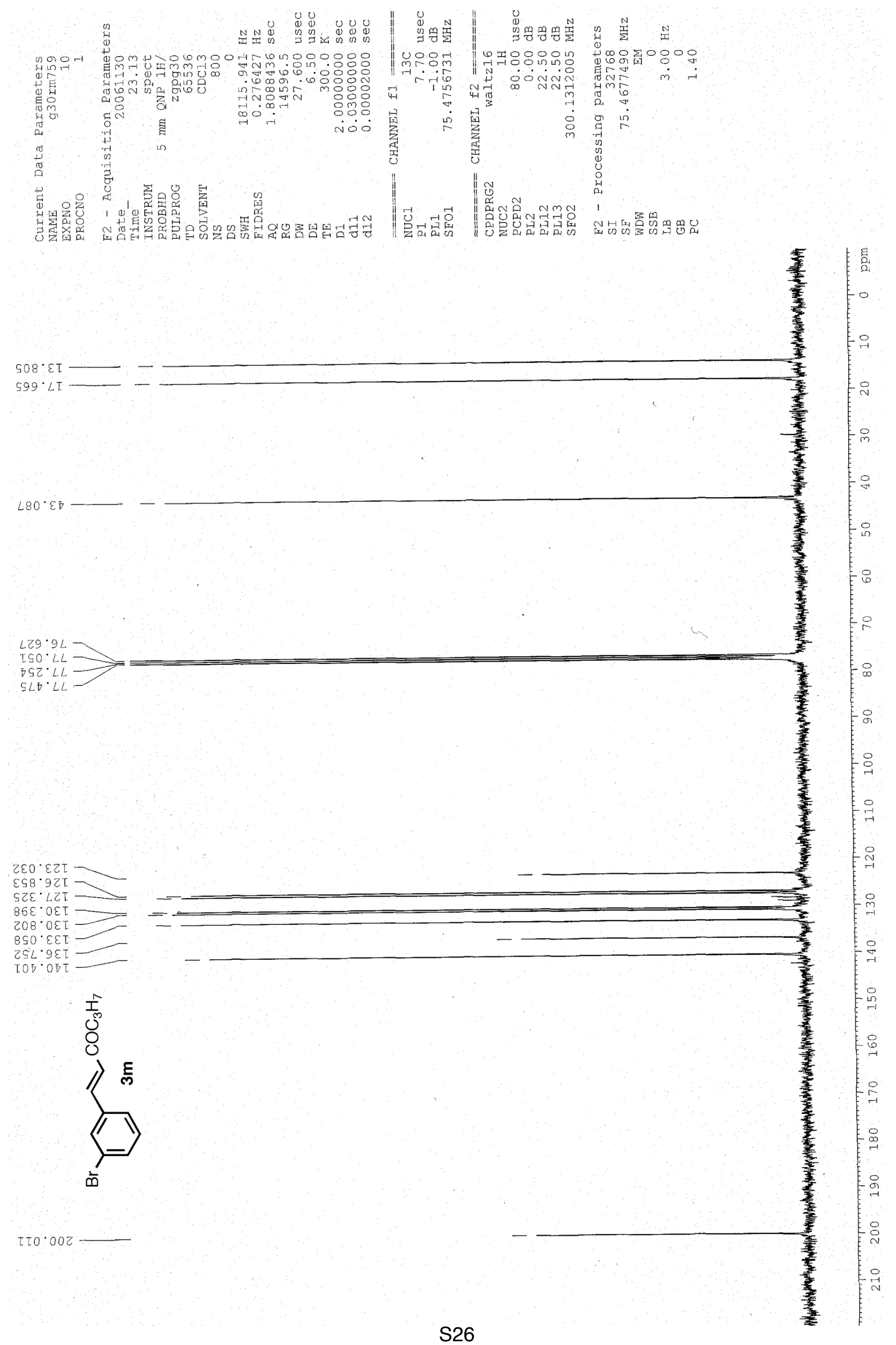

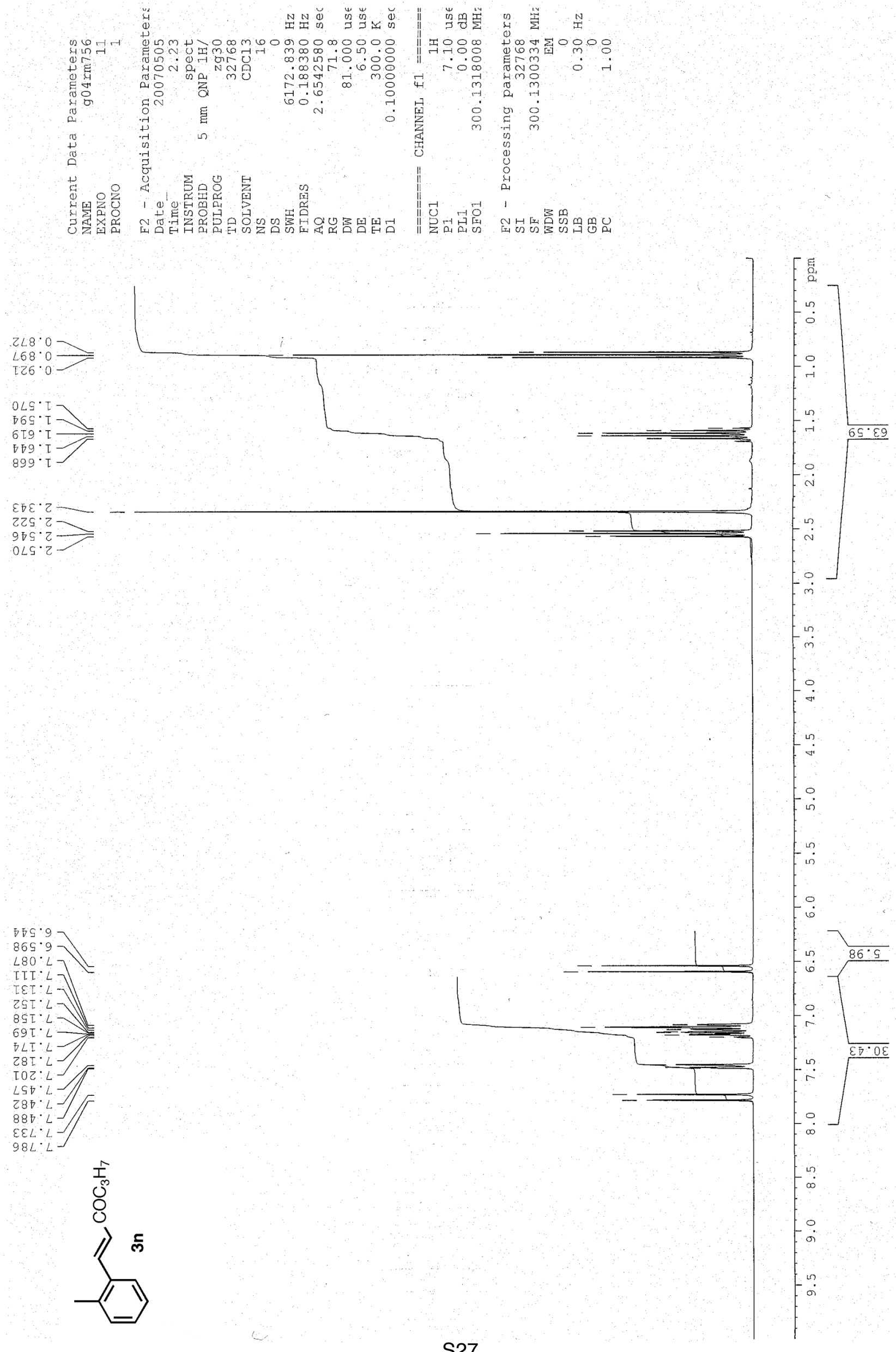

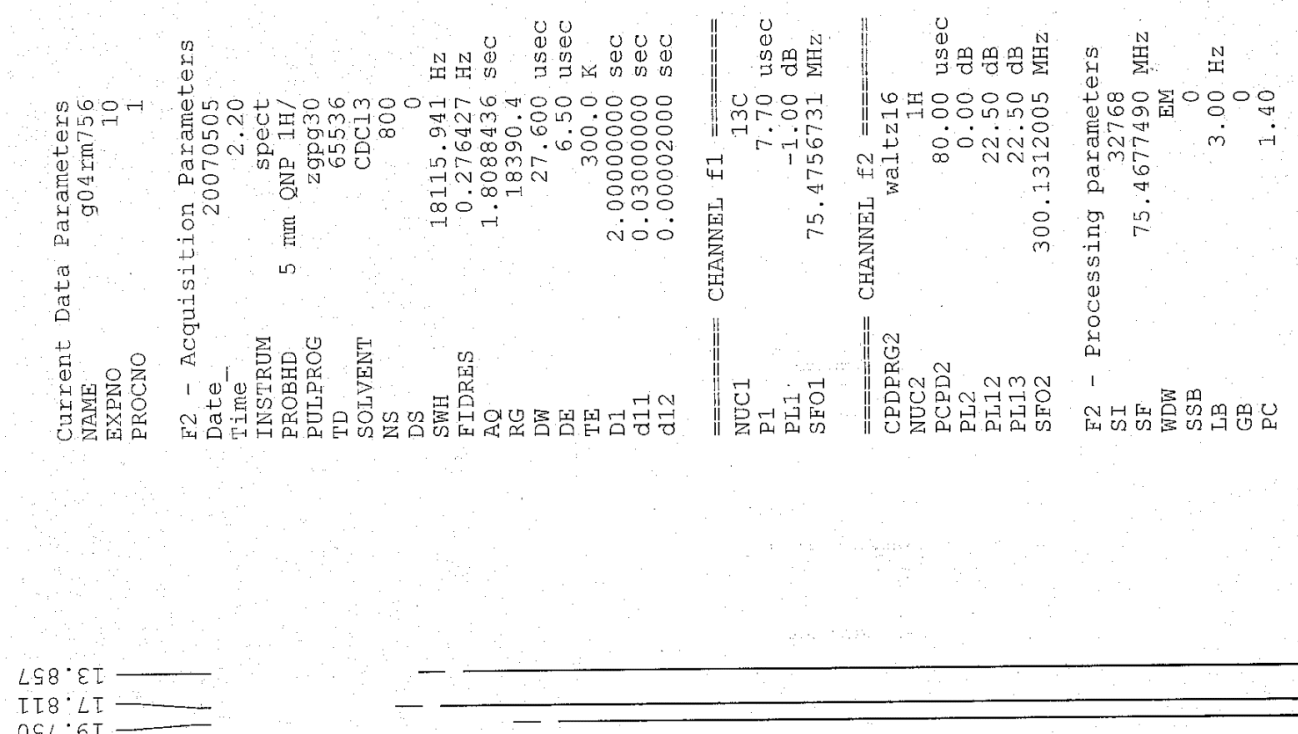

SOT'ยゅ
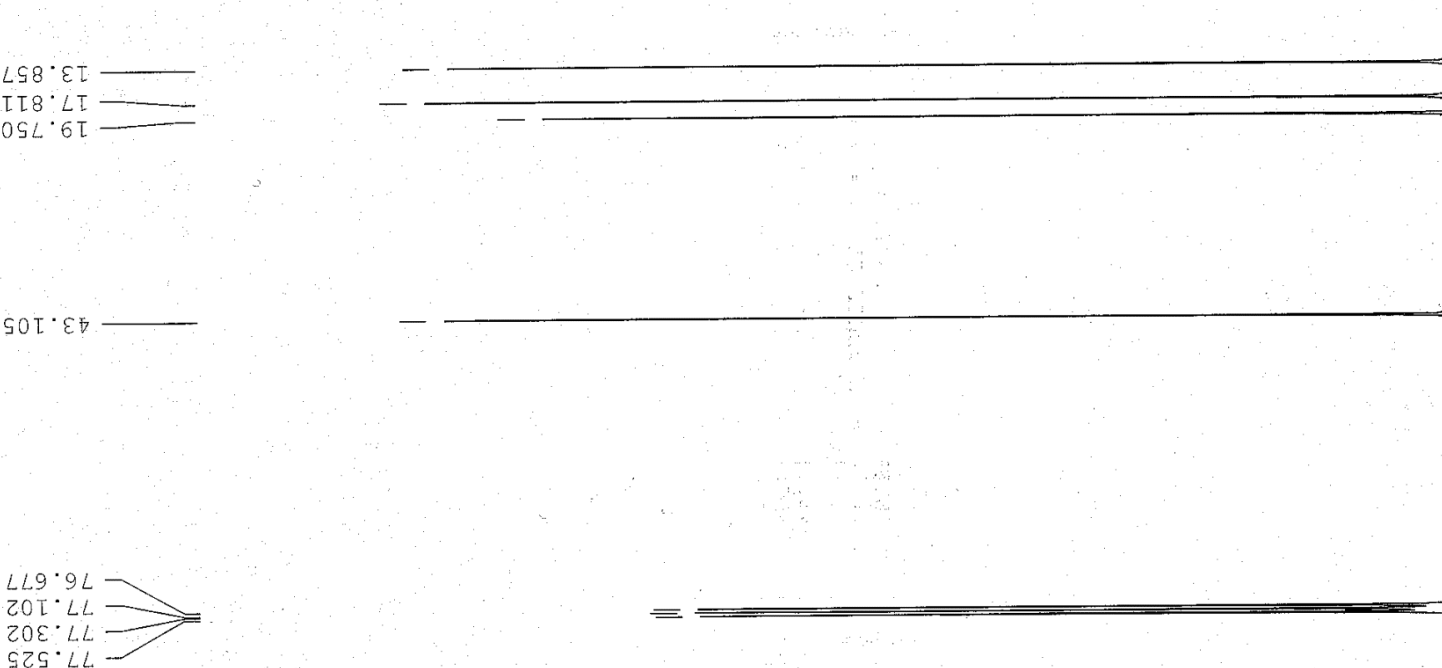

$\angle 0 \varepsilon^{\circ} 92 \tau$

गSE. $9 Z \mathrm{I} \longrightarrow$

$990.08 \mathrm{I}$

हso.0et

बक $\cdot \varepsilon \varepsilon$

$906 \cdot \operatorname{LET}$ -

$29 L 6 \varepsilon \mathrm{L}$

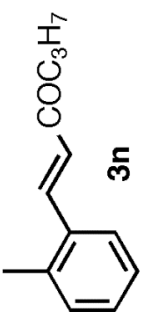

$$
-
$$

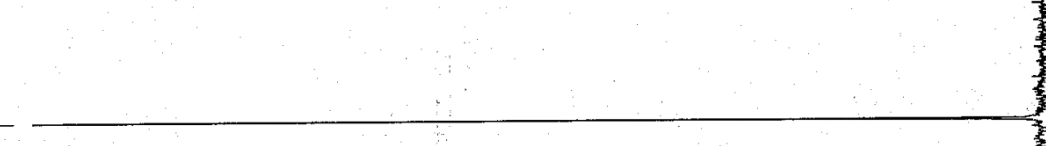



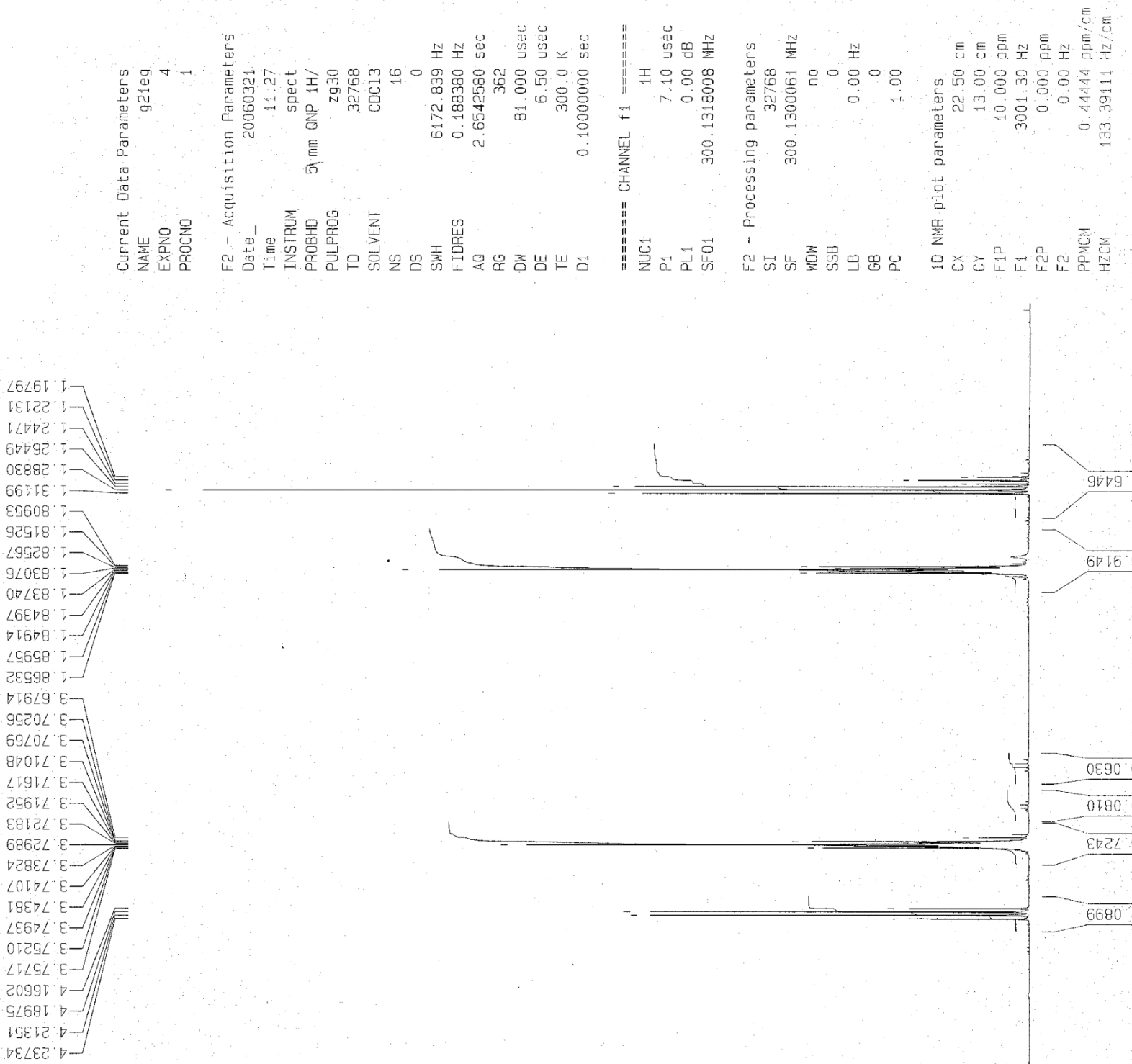

$\varepsilon 889 \angle \mathrm{L}-$

02800.5 $660 \angle 0.9-$ $\angle T 6 \angle 09$

$18 \angle 80.9-$

eEgol 9-

LEETI 9-

69ट2ट1 9

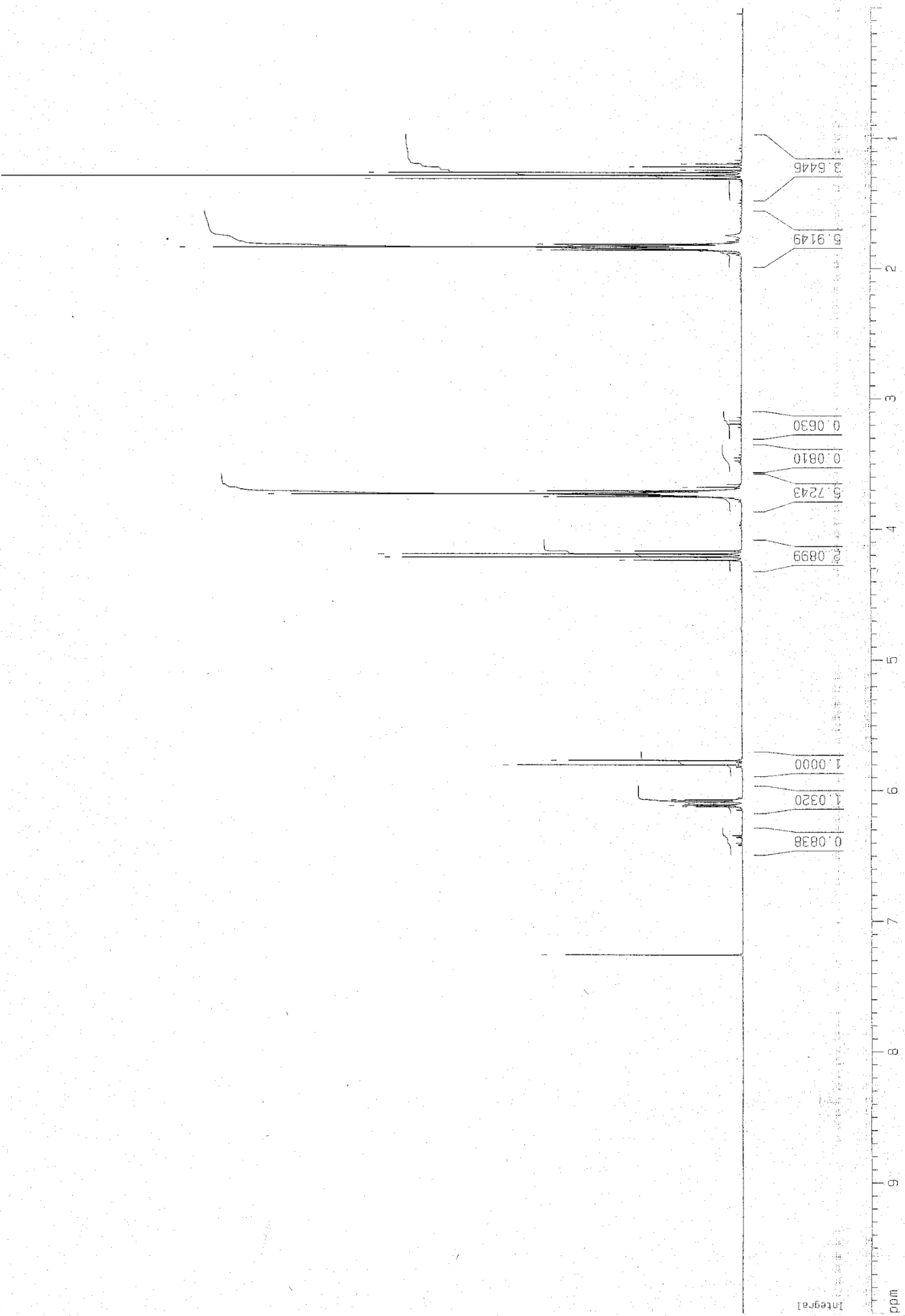




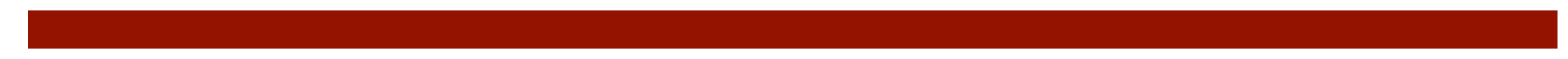

